\title{
AN ALTERNATING DIRECTION METHOD OF MULTIPLIERS FOR THE NUMERICAL SOLUTION OF A FULLY NONLINEAR PARTIAL DIFFERENTIAL EQUATION INVOLVING THE JACOBIAN DETERMINANT*
}

\author{
ALEXANDRE CABOUSSAT ${ }^{\dagger}$ AND ROLAND GLOWINSKI ${ }^{\ddagger}$
}

\begin{abstract}
We consider the Dirichlet problem for a partial differential equation involving the Jacobian determinant in two dimensions of space. The problem consists in finding a vector-valued function such that the determinant of its gradient is given pointwise in a bounded domain, together with essential boundary conditions. This problem was initially considered in Dacorogna and Moser [Ann. Inst. H. Poincaré Anal. Non Linéaire, 7 (1900), pp. 1-26], and several theoretical generalizations have been derived since. In this work, we design a numerical algorithm for the approximation of the solution of such a problem for various kinds of boundary data. The proposed method relies on an augmented Lagrangian algorithm with biharmonic regularization, and low order mixed finite element approximations. An iterative method allows us to decouple the nonlinearity and the differential operators. Numerical experiments show the capabilities of the method for benchmarks and then for more demanding test problems.
\end{abstract}

Key words. Jacobian determinant, augmented Lagrangian methods, ADMM algorithm, biharmonic regularization, finite element method, quadratically constrained minimization

AMS subject classifications. 65N30, 65K10, 49M20, 35F 30

DOI. $10.1137 / 16 \mathrm{M} 1094075$

1. Introduction. Numerical methods for fully nonlinear equations have received wide attention for the last decade and half. In particular, various approaches have been proposed for the numerical solution of second order equations (see, e.g., [9, 20, $21,22,24,27,33]$ ), including the Monge-Ampère equation, followed by other second order equations, such as Pucci's [10, 26, 27] or the curvature equations $[4,35,39,40]$. First order fully nonlinear equations, including the Eikonal equation $[7,16]$ or the Hamilton-Jacobi equation [37], have also been used in various mathematical models in science and engineering, e.g., in optics, wave propagation, material science, differential geometry (geodesics), geophysics, and image processing. Both classes of equations can be summarized into the prototypical form $F\left(\mathbf{u}, \nabla \mathbf{u}, \mathbf{D}^{2} \mathbf{u}, \mathbf{x}\right)=0$, for some function $F$ given, together with boundary conditions.

In this work, we focus on the Dirichlet problem for a particular equation involving the Jacobian of an unknown function. More precisely, inspired by [15, 17], and for a given datum $f$, we want to find $\mathbf{u}$ such that $\operatorname{det} \nabla \mathbf{u}=f$ in a bounded domain $\Omega$, together with Dirichlet boundary conditions. A first approach was proposed in [6] to validate the feasibility of the approach in the standard case $f=1$ and $u(\mathbf{x})=\mathbf{x}$ as Dirichlet boundary conditions. This work is the extension to more general, possibly

*Submitted to the journal's Methods and Algorithms for Scientific Computing section October 11, 2016; accepted for publication (in revised form) October 17, 2017; published electronically January $5,2018$.

http://www.siam.org/journals/sisc/40-1/M109407.html

Funding: This work was partially supported by the Swiss National Science Foundation (grant 165785) and the National Science Foundation (grant NSF DMS-0913982).

${ }^{\dagger}$ Haute Ecole de Gestion de Genève (Geneva School of Business Administration), University of Applied Sciences Western Switzerland (HES-SO), 1227 Carouge, Switzerland (alexandre. caboussat@hesge.ch, http://campus.hesge.ch/caboussata/).

${ }_{\ddagger}^{\ddagger}$ Department of Mathematics, University of Houston, Houston, TX 77204-3008 (roland@math. uh.edu, http://math.uh.edu/ roland/), and Hong-Kong Baptist University, Kowloon, Hong-Kong.

A52 
nonsmooth cases.

Several works in the literature have focused on the prescribed Jacobian equation, starting with the original article [17] that has been developed and extended in [11, 15, 36, 41]. Early regularity results for the determinant of the Jacobian of a given function have been developed in [13]. The goal of the present work is to provide an alternative, from the computational viewpoint, to the theoretical, explicit construction of solutions that exists in the literature for simple cases and to design a numerical method for the finite element approximation of the prescribed Jacobian equation for arbitrary two-dimensional domains

Following previous work on the Monge-Ampère equation [9], we advocate a variational approach for the solution of the prescribed Jacobian equation. Indeed, we introduce a cost function (typically a function of some distance to a given vectorvalued function, $\mathbf{x}$ for example) that we minimize over a set of vector-valued functions verifying the prescribed Jacobian equation as a nonlinear constraint. To solve the above variational problem, we employ an iterative method of the ADMM (for alternating direction method of multipliers) type: with this algorithm we will have to solve alternatively elliptic linear variational problems and nonlinearly constrained optimization problems which can be solved pointwise (in practice trianglewise if one uses triangulation-based finite element approximations, as done in this work). It is worth noticing that the local minimization problems we mentioned just above are four-dimensional, but they can be reduced to nonlinear one-dimensional problems that we solve by Newton's method.

The numerical validation is achieved first via the solution of simple test problems, allowing among other things a computational investigation of the convergence properties of our methodology. Next, we investigate the solution of more demanding test problems associated with nonsmooth data and/or nonconvex domains. Finally, we investigate the behavior of our iterative method when applied to the solution of problems without solution. Indeed these numerical experiments illustrate the accuracy and the robustness of the proposed computational methodology.

The solution of related problems from incompressible finite elasticity has been addressed in [31] (see also the references therein); for these problems, the incompressibility condition reads as $\operatorname{det}\left(\mathbf{I}_{d}+\nabla \mathbf{u}\right)=1, \mathbf{I}_{d}$ being the identity tensor and $\mathbf{u}$ a displacement field.

2. Problem formulation. Let $\Omega$ be a bounded domain of $\mathbb{R}^{2}$; we denote by $\Gamma$ the boundary of $\Omega$; let $f: \Omega \rightarrow \mathbb{R}$ and $\mathbf{g}: \Gamma \rightarrow \mathbb{R}^{2}$ be given functions. In the present work another assumption is made concerning $f$, namely

$$
f \geq 0 \quad \text { a.e. in } \Omega \text {. }
$$

The partial differential equation involving the Jacobian determinant (denoted in what follows by (JAC)) that we want to solve reads as follows: find $\mathbf{u}: \Omega \rightarrow \mathbb{R}^{2}$ satisfying

$$
\begin{cases}\operatorname{det} \nabla \mathbf{u}=f & \text { in } \Omega, \\ \mathbf{u}=\mathbf{g} & \text { on } \Gamma .\end{cases}
$$

In particular, we are interested in the identity boundary condition (i.e., $\mathbf{u}(\mathbf{x})=\mathbf{x}$ for $\mathbf{x} \in \Gamma)$ that has been considered in $[6]$, and in $[11,17]$ from a theoretical point of view, 
that is for problems of the following type:

$$
\begin{cases}\operatorname{det} \nabla \mathbf{u}=f & \text { in } \Omega \\ \mathbf{u}(\mathbf{x})=\mathbf{x} & \text { a.e. } \mathbf{x} \text { on } \Gamma\end{cases}
$$

Note that the case when $f$ has enough regularity $\left(f \in C^{0, \alpha}(\Omega)\right.$ for some $\left.\alpha \geq 0\right)$ has been addressed in [17]; we will also consider numerically right-hand sides with less regularity (typically $f \in L^{p}(\Omega)$ for some $p \geq 0$ ). Problem (3) corresponds to finding a mapping $\mathbf{u}$ that preserves both the boundary data and some kind of volume (up to some stretching of the mapping). Note that the solution to (3) is not necessarily unique (and a fortiori, the same remark holds for (2)). Indeed, let us consider (3) with $f=1$ and $\Omega$ the unit disk centered at the origin; in this case, $\mathbf{u}(\mathbf{x})=\mathbf{x}$ is an obvious solution. However, when using the polar coordinates $(\rho, \theta)$, one can see that $\mathbf{v}$ defined by $\mathbf{v}(\rho, \theta)=\left(\rho \cos \left(\theta+2 k \pi \rho^{2}\right), \rho \sin \left(\theta+2 k \pi \rho^{2}\right)\right)^{T}$ is also a solution.

The proof of existence of a solution to (2) (via the divergence theorem) requires data to be compatible with the geometrical domain [17]. When the boundary conditions are given by $\mathbf{u}(\mathbf{x})=\mathbf{x}$ on $\Gamma$, this compatibility condition reads as

$$
\int_{\Omega} f d \mathbf{x}=\text { measure }(\Omega)
$$

Condition (1) is useful from an analytical point of view to prove existence results; however, it has been recently loosened (slightly) to accept locally negative data (see [15] for details). Condition (1) makes problem (2) elliptic, an important feature for the solution methodology discussed in this work. From now on we will assume that both assumptions (4) and (1) hold; however, in sections 5 and 6 , we will study computationally the behavior of the solution methods investigated in the present work when these conditions are not satisfied.

Remark 2.1. After suitable transformations, one can show (see [17]) that (2) is equivalent to the following inverse divergence problem: find $\mathbf{v}: \Omega \rightarrow \mathbb{R}$ satisfying

$$
\begin{cases}\nabla \cdot \mathbf{v}=f-1 & \text { in } \Omega \\ \mathbf{v}=\mathbf{0} & \text { on } \Gamma .\end{cases}
$$

This problem has been studied in [1,2], and the authors of the present work have proposed in [5] a numerical method for the solution of a closely related problem.

In order to enforce the uniqueness of the solution to (2), we use a variational approach relying on the following constrained minimization problem:

$$
\min _{\mathbf{v} \in E} J(\mathbf{v})
$$

where

$$
J(\mathbf{v})=\frac{1}{2} \int_{\Omega}\left|\nabla \mathbf{v}-\mathbf{I}_{d}\right|^{2} d \mathbf{x}
$$

and

$$
E=\left\{\mathbf{v} \in H^{1}(\Omega)^{2}, \operatorname{det} \nabla \mathbf{v}=f,\left.\mathbf{v}\right|_{\Gamma}=\mathbf{g}\right\} ;
$$

here, $\mathbf{I}_{d}$ denotes the $2 \times 2$ identity matrix (other functionals than the one in (7) may be used). From now on, we will denote by $\mathbf{u}$ the solution to (6). The Frobenius norm 
and inner product are respectively defined by $|\mathbf{T}|=(\mathbf{T}: \mathbf{T})^{1 / 2}, \mathbf{S}: \mathbf{T}=\sum_{i, j=1}^{2} s_{i j} t_{i j}$ for every $\mathbf{S}=\left(s_{i j}\right), \mathbf{T}=\left(t_{i j}\right) \in \mathbb{R}^{2 \times 2}$. The choice of the objective function is made in order to find the solution that is the closest to the identity function $\mathbf{x} \rightarrow \mathbf{x}$, which corresponds to the least deformation inside the domain. It is not necessarily compatible with the boundary conditions, which may lead to a nonzero value of the objective function even at optimum, but it still allows us to enforce the uniqueness of the solution (see also Remark 2.2).

Remark 2.2. As stated before, the choice of the term $\mathbf{I}_{d}$ in the objective function (7) to enforce uniqueness is actually arbitrary. It could be replaced with another tensor-valued function (including the zero tensor) without modifying the proposed algorithm.

Remark 2.3. The term $\mathbf{I}_{d}$ in the objective function could be replaced by the gradient of an harmonic extension of the boundary data $g$ into the domain $\Omega$. This choice would increase the smoothness (in the neighborhood of the boundary) of the minimiser "chosen" by the algorithm.

\section{Augmented Lagrangian framework and iterative algorithm.}

3.1. Regularization and augmented Lagrangian approach. First we introduce a biharmonic regularization of the variational problem (6). This regularization of our problem by introducing a higher order operator is reminiscent of viscosity solutions $[14,32]$. Let us consider a parameter $\delta>0$. The biharmonic regularized problem reads as

$$
\min _{\mathbf{v} \in \tilde{E}}\left[\frac{1}{2} \int_{\Omega}\left|\nabla \mathbf{v}-\mathbf{I}_{d}\right|^{2} d \mathbf{x}+\frac{\delta}{2} \int_{\Omega}\left|\nabla^{2} \mathbf{v}\right|^{2} d \mathbf{x}\right]
$$

with

$$
\tilde{E}=\left\{\mathbf{v} \in H^{2}(\Omega)^{2}, \operatorname{det} \nabla \mathbf{v}=f,\left.\mathbf{v}\right|_{\Gamma}=\mathbf{g}\right\} .
$$

Let us introduce $\mathbf{p} \in L^{2}(\Omega)^{2 \times 2}$ and relax (9) by introduction of an auxiliary variable. Thus, (9) becomes equivalent to

$$
\min _{(\mathbf{v}, \mathbf{q}) \in \hat{E}}\left[\frac{1}{2} \int_{\Omega}\left|\nabla \mathbf{v}-\mathbf{I}_{d}\right|^{2} d \mathbf{x}+\frac{\delta}{2} \int_{\Omega}\left|\nabla^{2} \mathbf{v}\right|^{2} d \mathbf{x}\right]
$$

with

$$
\hat{E}=\left\{(\mathbf{v}, \mathbf{q}) \in H^{2}(\Omega)^{2} \times L^{2}(\Omega)^{2 \times 2}, \operatorname{det} \mathbf{q}=f,\left.\mathbf{v}\right|_{\Gamma}=\mathbf{g}, \nabla \mathbf{v}=\mathbf{q}\right\} .
$$

Remark 3.1. Since $\Omega$ is bounded in $\mathbb{R}^{2}$, it follows from the Sobolev imbedding theorem (see, e.g., [34]) that

$$
\mathbf{v} \in H^{2}(\Omega)^{2} \Rightarrow \nabla \mathbf{v} \in H^{1}(\Omega)^{2 \times 2} \Rightarrow \operatorname{det} \nabla \mathbf{v} \in W^{1, s}(\Omega), \quad \forall s \in[1,2)
$$

and that

$$
\left.\mathbf{v}\right|_{\Gamma} \in H^{3 / 2}(\Gamma)^{2} .
$$

It follows from the above properties that $f \in W^{1,2}(\Omega)$ and $\mathbf{g} \in H^{3 / 2}(\Gamma)^{2}$ are necessary (but not sufficient) conditions for $\tilde{E}$ and $\hat{E}$ to be nonempty. 
Remark 3.2. The addition of the biharmonic regularization term in (9) requires the solution to be in $H^{2}(\Omega)^{2}$. However, the solution to (6) has the $H^{1}(\Omega)^{2}$-regularity. Following this initial requirement, the additional variable $\mathbf{p}$ has to have the $L^{2}(\Omega)^{2 \times 2}$ regularity. From the discrete point of view, the design of the mixed finite element method does not depend on this initial choice at the continuous level.

We advocate an augmented Lagrangian algorithm for the solution of (11). Namely, for $r>0$ a given parameter, we want to find a saddle-point of the augmented Lagrangian functional

$$
\begin{aligned}
\mathcal{L}(\mathbf{v}, \mathbf{q} ; \boldsymbol{\mu})= & \frac{1}{2} \int_{\Omega}\left|\nabla \mathbf{v}-\mathbf{I}_{d}\right|^{2} d \mathbf{x}+\frac{\delta}{2} \int_{\Omega}\left|\nabla^{2} \mathbf{v}\right|^{2} d \mathbf{x} \\
& +\frac{r}{2} \int_{\Omega}|\nabla \mathbf{v}-\mathbf{q}|^{2} d \mathbf{x}+\int_{\Omega} \boldsymbol{\mu}:(\nabla \mathbf{v}-\mathbf{q}) d \mathbf{x} .
\end{aligned}
$$

More precisely, let us define the function spaces

$$
\begin{aligned}
\mathbf{V}_{\mathbf{g}} & =\left\{\mathbf{v} \in H^{2}(\Omega)^{2},\left.\mathbf{v}\right|_{\Gamma}=\mathbf{g}\right\}, \\
\mathbf{Q} & =L^{2}(\Omega)^{2 \times 2}, \\
\mathbf{Q}_{f} & =\{\mathbf{q} \in \mathbf{Q}, \operatorname{det} \mathbf{q}=f\} ;
\end{aligned}
$$

then our goal is to find $\{\mathbf{u}, \mathbf{p}, \boldsymbol{\lambda}\} \in \mathbf{V}_{\mathbf{g}} \times \mathbf{Q}_{f} \times \mathbf{Q}$ such that

$$
\mathcal{L}(\mathbf{u}, \mathbf{p} ; \boldsymbol{\mu}) \leq \mathcal{L}(\mathbf{u}, \mathbf{p} ; \boldsymbol{\lambda}) \leq \mathcal{L}(\mathbf{v}, \mathbf{q} ; \boldsymbol{\lambda})
$$

for all $\{\mathbf{v}, \mathbf{q}, \boldsymbol{\mu}\} \in \mathbf{V}_{\mathbf{g}} \times \mathbf{Q}_{f} \times \mathbf{Q}$ since, if (17) holds, $\mathbf{u}$ is a solution of problem (11) and $\mathbf{p}=\nabla \mathbf{u}$.

Sufficient conditions for the existence of a multiplier $\lambda$ for the augmented Lagrangian function (13) can be found in $[19,23]$. For the finite dimensional discretized problem, since the constraints are linear $\left(\nabla \mathbf{v}_{h}=\mathbf{q}_{h}\right)$ and with sufficient regularity, the constraint qualification for equality constraint holds.

3.2. A primal-dual algorithm. In order to solve the saddle-point problem (17) we advocate a primal-dual algorithm belonging to the ADMM (for alternating direction method of multipliers) family. To the best of our knowledge, this type of method has been introduced in [28, 29] and further discussed and applied to a large variety of convex and nonconvex variational problems in, e.g., [3, 23, 25, 27, 31] (see also the many references therein).

In order to apply such an algorithm, let $\left(\mathbf{u}^{0}, \boldsymbol{\lambda}^{0}\right)$ be given in $\mathbf{V}_{\mathbf{g}} \times \mathbf{Q}$. Then, for $n \geq 1,\left(\mathbf{u}^{n-1}, \boldsymbol{\lambda}^{n-1}\right)$ being known, we compute $\mathbf{p}^{n}, \mathbf{u}^{n}$, and $\boldsymbol{\lambda}^{n}$ as follows:

1. To obtain $\mathbf{p}^{n}$, solve the nonlinearly constrained minimization problem

$$
\mathbf{p}^{n}=\arg \min _{\mathbf{q} \in \mathbf{Q}_{f}} \mathcal{L}\left(\mathbf{u}^{n-1}, \mathbf{q} ; \boldsymbol{\lambda}^{n-1}\right),
$$

which is equivalent to

$$
\mathbf{p}^{n}=\arg \min _{\mathbf{q} \in \mathbf{Q}_{f}}\left[\frac{r}{2} \int_{\Omega}|\mathbf{q}|^{2} d \mathbf{x}-\int_{\Omega} \mathbf{X}^{n-1}: \mathbf{q} d \mathbf{x}\right],
$$

where $\mathbf{X}^{n}:=r \nabla \mathbf{u}^{n}+\boldsymbol{\lambda}^{n} \in \mathbf{Q}$. Since problem (18) does not involve derivatives of $\mathbf{q}$, it can be solved pointwise (in practice, for example, for each element of 
a finite element triangulation). It reads, namely, as follows: for a.e. $\mathbf{x} \in \Omega$, find $\mathbf{p}^{n}(\mathbf{x}) \in \mathbb{R}^{2 \times 2}$ verifying

$$
\mathbf{p}^{n}(\mathbf{x})=\arg \min _{\mathbf{q} \in \mathbf{Q}_{\mathbf{x}}}\left[\frac{r}{2}|\mathbf{q}|^{2}-\mathbf{X}^{n-1}(\mathbf{x}): \mathbf{q}\right]
$$

with

$$
\mathbf{Q}_{\mathbf{x}}=\left\{\mathbf{q} \in \mathbb{R}^{2 \times 2}, \operatorname{det} \mathbf{q}=q_{11} q_{22}-q_{12} q_{21}=f(\mathbf{x})\right\} .
$$

2. To obtain $\mathbf{u}^{n}$, solve the linear variational problem

$$
\mathbf{u}^{n}=\arg \min _{\mathbf{v} \in \mathbf{V}_{\mathbf{g}}} \mathcal{L}\left(\mathbf{v}, \mathbf{p}^{n} ; \boldsymbol{\lambda}^{n-1}\right),
$$

which is, more explicitly,

$$
\mathbf{u}^{n}=\arg \min _{\mathbf{v} \in \mathbf{V}_{\mathbf{g}}}\left[\frac{\delta}{2} \int_{\Omega}\left|\nabla^{2} \mathbf{v}\right|^{2} d \mathbf{x}+\frac{1+r}{2} \int_{\Omega}|\nabla \mathbf{v}|^{2} d \mathbf{x}-\int_{\Omega} \nabla \mathbf{v}: \mathbf{Y}^{n} d \mathbf{x}\right],
$$

where $\mathbf{Y}^{n}:=\mathbf{I}_{d}+r \mathbf{p}^{n}-\boldsymbol{\lambda}^{n-1} \in \mathbf{Q}$. Written in variational form, the EulerLagrange equation associated with the minimization problem (21) reads as follows: find $\mathbf{u} \in \mathbf{V}_{\mathbf{g}}$ satisfying

$$
\delta \int_{\Omega} \nabla^{2} \mathbf{u} \cdot \nabla^{2} \mathbf{v} d \mathbf{x}+(1+r) \int_{\Omega} \nabla \mathbf{u}: \nabla \mathbf{v} d \mathbf{x}=\int_{\Omega} \mathbf{Y}^{n}: \nabla \mathbf{v} d \mathbf{x}
$$

for all $\mathbf{v} \in\left(H^{2}(\Omega) \cap H_{0}^{1}(\Omega)\right)^{2}$. Assuming that $\mathbf{g} \in H^{3 / 2}(\Omega)^{2}$, the linear variational problem (22) is well-posed.

3. To obtain $\boldsymbol{\lambda}^{n} \in \mathbf{Q}$, update $\boldsymbol{\lambda}^{n-1}$ via

$$
\lambda^{n}=\boldsymbol{\lambda}^{n-1}+r\left(\nabla \mathbf{u}^{n}-\mathbf{p}^{n}\right) .
$$

3.3. On the numerical solution of the local nonlinearly constrained minimization problems. Problem (19), namely

$$
\mathbf{p}^{n}(\mathbf{x})=\arg \min _{\mathbf{q} \in \mathbf{Q}_{\mathbf{x}}}\left[\frac{r}{2}|\mathbf{q}|^{2}-\mathbf{X}^{n-1}: \mathbf{q}\right]
$$

with $\mathbf{Q}_{\mathbf{x}}=\left\{\mathbf{q} \in \mathbb{R}^{2 \times 2}, \operatorname{det} \mathbf{q}=q_{11} q_{22}-q_{12} q_{21}=f(\mathbf{x})\right\}$, is clearly of low finite dimension (dimension 4 in fact). After division by $r$ and dropping of the superscript $n$, problem (19) becomes a particular case of the following nonlinear constrained minimization problem in $\mathbb{R}^{4}$ (after suitable renumbering of the variables):

$$
\min _{\mathbf{q} \in \mathbf{E}_{c}}\left[\frac{1}{2}|\mathbf{q}|^{2}-\mathbf{b} \cdot \mathbf{q}\right]
$$

with $\mathbf{E}_{c}=\left\{\mathbf{q} \in \mathbb{R}^{4}, q_{1} q_{2}-q_{3} q_{4}=c(>0)\right\}$. Actually, here, $c=f(\mathbf{x})$ and $\mathbf{b}=$ $\frac{1}{r}\left(\mathbf{X}_{11}^{n-1}, \mathbf{X}_{22}^{n-1}, \mathbf{X}_{12}^{n-1}, \mathbf{X}_{21}^{n-1}\right)$. Let us denote by $\mathbf{S}$ the $4 \times 4$ orthogonal matrix

$$
\mathbf{S}=\left(\begin{array}{cccc}
1 / \sqrt{2} & 1 / \sqrt{2} & 0 & 0 \\
1 / \sqrt{2} & -1 / \sqrt{2} & 0 & 0 \\
0 & 0 & 1 / \sqrt{2} & 1 / \sqrt{2} \\
0 & 0 & 1 / \sqrt{2} & -1 / \sqrt{2}
\end{array}\right)
$$

Copyright (c) by SIAM. Unauthorized reproduction of this article is prohibited. 
We then introduce the new variables $\mathbf{z}=\mathbf{S q}$ and $\boldsymbol{\beta}=\mathbf{S b}$. The minimization problem in (24) is then equivalent to

$$
\min _{\mathbf{z} \in \mathbf{F}_{c}}\left[\frac{1}{2}|\mathbf{z}|^{2}-\boldsymbol{\beta} \cdot \mathbf{z}\right]
$$

with $\mathbf{F}_{c}=\left\{\mathbf{z} \in \mathbb{R}^{4}, z_{1}^{2}-z_{2}^{2}-z_{3}^{2}+z_{4}^{2}=2 c(>0)\right\}$. Actually this problem arises also in incompressible finite elasticity (see, e.g., [31]); in order to solve it, let us thus introduce the associated Lagrangian functional

$$
L(\mathbf{z}, \mu)=\frac{1}{2}|\mathbf{z}|^{2}-\boldsymbol{\beta} \cdot \mathbf{z}-\frac{\mu}{2}\left(z_{1}^{2}-z_{2}^{2}-z_{3}^{2}+z_{4}^{2}-2 c\right) .
$$

If $\mathbf{y}$ is a solution of (26), and $\lambda$ is a related Lagrange multiplier, the first order optimality conditions read as follows:

$$
\begin{aligned}
y_{1}-\beta_{1} & =\lambda y_{1}, \\
y_{2}-\beta_{2} & =-\lambda y_{2}, \\
y_{3}-\beta_{3} & =-\lambda y_{3}, \\
y_{4}-\beta_{4} & =\lambda y_{4}, \\
y_{1}^{2}-y_{2}^{2}-y_{3}^{2}+y_{4}^{2} & =2 c .
\end{aligned}
$$

An alternative formulation of the optimality conditions is

$$
\begin{aligned}
y_{1} & =\frac{\beta_{1}}{1-\lambda}, \\
y_{2} & =\frac{\beta_{2}}{1+\lambda}, \\
y_{3} & =\frac{\beta_{3}}{1+\lambda}, \\
y_{4} & =\frac{\beta_{4}}{1-\lambda}, \\
\frac{\beta_{1}^{2}+\beta_{4}^{2}}{(1-\lambda)^{2}}-\frac{\beta_{2}^{2}+\beta_{3}^{2}}{(1+\lambda)^{2}} & =2 c .
\end{aligned}
$$

It can be shown (see, e.g., [38]) that the solution of (26) corresponds to the unique solution of

$$
\frac{\beta_{1}^{2}+\beta_{4}^{2}}{(1-\lambda)^{2}}-\frac{\beta_{2}^{2}+\beta_{3}^{2}}{(1+\lambda)^{2}}=2 c
$$

belonging to the interval $(-1,+1)$. In order to obtain a numerically well-posed problem solvable by a Newton method, we rewrite (28) as

$$
1-\lambda=\sqrt{\frac{\beta_{1}^{2}+\beta_{4}^{2}}{\frac{\beta_{2}^{2}+\beta_{3}^{2}}{(1+\lambda)^{2}}+2 c}}
$$

or, equivalently,

$$
\phi(\lambda):=1-\lambda-\frac{(1+\lambda) \sqrt{\beta_{1}^{2}+\beta_{4}^{2}}}{\sqrt{\beta_{2}^{2}+\beta_{3}^{2}+2 c(1+\lambda)^{2}}}=0 .
$$

Problem (30) is solved by a Newton method with initial guess $\lambda^{0}=0$. 
Remark 3.3. Actually, direct calculations for the implementation of the Newton method show that

$$
\phi^{\prime}(\lambda)=-1-\frac{\sqrt{\beta_{1}^{2}+\beta_{4}^{2}}}{F^{2}}\left(F-\frac{2 c(1+\lambda)^{2}}{F}\right)
$$

with $F=\sqrt{\beta_{2}^{2}+\beta_{3}^{2}+2 c(1+\lambda)^{2}}$. The numerical experiments always show convergence to a root in $(-1,+1)$, except for the first iterate of the outer iterative algorithm, when the initial data are far from the solution. However, this lack of convergence during the transient phase does not jeopardize the global convergence of the ADMM algorithm; indeed, good convergence properties are recovered after a few outer iterations.

3.4. Numerical solution of the linear variational problems. The first order optimality conditions related to (21) are the following: find $\mathbf{u}^{n+1} \in \mathbf{V}_{\mathbf{g}}$ such that

$$
\delta \int_{\Omega} \nabla^{2} \mathbf{u}^{n+1} \cdot \nabla^{2} \mathbf{v} d \mathbf{x}+(1+r) \int_{\Omega} \nabla \mathbf{u}^{n+1}: \nabla \mathbf{v} d \mathbf{x}=\int_{\Omega} \mathbf{Y}^{n}: \nabla \mathbf{v} d \mathbf{x}
$$

for all $\mathbf{v} \in \mathbf{V}_{0}$, where $\mathbf{V}_{0}=\left\{\mathbf{v} \in H^{2}(\Omega)^{2},\left.\mathbf{v}\right|_{\Gamma}=\mathbf{0}\right\}$. Problem (31) is a classical biharmonic problem. Note that biharmonic problems closely related to (31) have been encountered when solving the elliptic Monge-Ampère equation in [9]. We observe that this problem is equivalent (if $\Omega$ is convex or $\Gamma$ smooth enough) to the following second order variational system:

$$
\left\{\begin{array}{l}
\mathbf{w}^{n+1} \in\left(H_{0}^{1}(\Omega)\right)^{2}, \\
\delta \int_{\Omega} \nabla \mathbf{w}^{n+1}: \nabla \mathbf{v} d \mathbf{x}+(1+r) \int_{\Omega} \mathbf{w}^{n+1} \cdot \mathbf{v} d \mathbf{x}=\int_{\Omega} \mathbf{Y}^{n}: \nabla \mathbf{v} d \mathbf{x} \\
\forall \mathbf{v} \in\left(H_{0}^{1}(\Omega)\right)^{2}
\end{array}\right.
$$

together with

$$
\left\{\begin{array}{l}
\mathbf{u}^{n+1} \in\left(H^{1}(\Omega)\right)^{2},\left.\quad \mathbf{u}^{n+1}\right|_{\partial \Omega}=\mathbf{g}, \\
\int_{\Omega} \nabla \mathbf{u}^{n+1}: \nabla \mathbf{v} d \mathbf{x}=\int_{\Omega} \mathbf{w}^{n+1} \cdot \mathbf{v} d \mathbf{x} \\
\forall \mathbf{v} \in\left(H_{0}^{1}(\Omega)\right)^{2}
\end{array}\right.
$$

Both problems (32) and (33) are nothing but well-posed linear second order elliptic problems formulated in a variational way. Nowadays, the solution of such problems is routine: if, for example, one uses well-chosen finite element approximations of problems (32) and (33) (an issue to be addressed in section 4), the resulting finite dimensional problems are linear systems associated with matrices which are symmetric positive definite and sparse; from these properties, sparse Cholesky solvers are obvious candidates for the solution of the discrete analogues of problems (32) and (33).

4. Finite element approximation. The methodology described in sections 2 and 3 has a variational nature; it therefore makes perfect sense to use a finite elementbased methodology to implement it. Indeed, the finite element approximation that we are going to use is closely related to those discussed in, e.g., $[9,25,27]$ for the solution of closely related biharmonic problems. To approximate the variable $\mathbf{v}$ (respectively, q) we will make use of finite element spaces of vector-valued (respectively, 
$2 \times 2$ tensor-valued) functions, globally continuous and piecewise affine (respectively, possibly discontinuous and piecewise constant) over a triangulation; the spaces used to approximate $\mathbf{q}$ will also be used to approximate $\nabla \mathbf{v}$ and the Lagrange multipliers. Such finite element spaces are described below.

Let $h>0$ be a space discretization step. A family $\left\{\Omega_{h}\right\}_{h}$ of polygonal approximations of the domain $\Omega$ is introduced such that $\lim _{h \rightarrow 0} \Omega_{h}=\Omega$, together with $\lim _{h \rightarrow 0} \Gamma_{h}=\Gamma$ (if $\Omega$ is polygonal, we take $\Omega_{h}=\Omega$ for all $h$ ). We consider a family $\left\{\mathcal{T}_{h}\right\}_{h}$ of conforming triangulations of $\Omega_{h}$, verifying the classical assumptions (see, e.g., [25, Appendix 1]). From $\mathcal{T}_{h}$, we approximate the spaces $\mathbf{Q}$ and $\mathbf{Q}_{f}$, respectively, by

$$
\mathbf{Q}_{h}=\left\{\mathbf{q} \in L^{2}(\Omega)^{2 \times 2},\left.\mathbf{q}\right|_{T} \in \mathbb{R}^{2 \times 2}, \forall T \in \mathcal{T}_{h}\right\}
$$

and

$$
\mathbf{Q}_{f h}=\left\{\mathbf{q} \in \mathbf{Q}_{h},\left.\operatorname{det} \mathbf{q}\right|_{T}=\bar{f}_{T}, \forall T \in \mathcal{T}_{h}\right\},
$$

where, for all $T \in \mathcal{T}_{h}, \bar{f}_{T}=\frac{1}{|T|} \int_{T} f(\mathbf{x}) d \mathbf{x},|T|$ being the measure of $T$ (if $f$ is continuous over $\bar{T}$, we advocate the trapezoidal rule to approximate the above integral). On the other hand, we approximate the space $\mathbf{V}_{\mathbf{g}}$ by

$$
\mathbf{V}_{\mathbf{g}, h}=\left\{\mathbf{v} \in C^{0}\left(\overline{\Omega_{h}}\right)^{2},\left.\mathbf{v}\right|_{T} \in\left(\mathbb{P}_{1}\right)^{2}, \forall T \in \mathcal{T}_{h},\left.\mathbf{v}\right|_{\Gamma_{h}}=\mathbf{g}_{h}\right\}
$$

with $\mathbb{P}_{1}$ the space of the two-variable polynomials of degree $\leq 1$ and $\mathbf{g}_{h}$ an approximation of $\mathbf{g}$ (if $\mathbf{g}$ is continuous, which is definitely the case if $\mathbf{g} \in H^{3 / 2}(\Gamma)^{2}$, one should replace the boundary condition $\left.\mathbf{v}\right|_{\Gamma_{h}}=\mathbf{g}_{h}$ by $\mathbf{v}(P)=\mathbf{g}(P)$ for all the vertices $P \in \mathcal{T}_{h}$ belonging to $\Gamma_{h}$; this supposes that the vertices of $\mathcal{T}_{h}$ belonging to $\Gamma_{h}$ also belong to $\Gamma$, an assumption we will ever make). To approximate the Sobolev spaces $H_{0}^{1}(\Omega)^{2}$ and $\left(H^{2}(\Omega) \cap H_{0}^{1}(\Omega)\right)^{2}$ we will employ $\mathbf{V}_{0 h}$ defined by

$$
\mathbf{V}_{0 h}=\left\{\mathbf{v} \in C^{0}\left(\overline{\Omega_{h}}\right)^{2},\left.\mathbf{v}\right|_{T} \in\left(\mathbb{P}_{1}\right)^{2}, \forall T \in \mathcal{T}_{h},\left.\mathbf{v}\right|_{\Gamma_{h}}=\mathbf{0}\right\} .
$$

We equip $\mathbf{Q}_{h}$ (and $\mathbf{Q}_{f h}$ ) with a discrete inner product and norm, respectively, defined by

$$
((\mathbf{p}, \mathbf{q}))_{0 h}=\left.\sum_{T \in \mathcal{T}_{h}}|T| \mathbf{p}\right|_{T}:\left.\mathbf{q}\right|_{T}, \quad|||\mathbf{q}| \|_{0 h}^{2}=\sqrt{((\mathbf{q}, \mathbf{q}))_{0 h}}
$$

Similarly, we equip $\mathbf{V}_{\mathbf{g}, h}$ (and $\mathbf{V}_{0 h}$ ) with a discrete inner product and norm, respectively, defined by

$$
(\mathbf{u}, \mathbf{v})_{0 h}=\frac{1}{3} \sum_{T \in \mathcal{T}_{h}}|T| \sum_{j=1}^{3} \mathbf{u}\left(P_{T_{j}}\right) \cdot \mathbf{v}\left(P_{T_{j}}\right), \quad\|\mathbf{u}\|_{0 h}^{2}=\sqrt{(\mathbf{u}, \mathbf{u})_{0 h}}
$$

$P_{T_{1}}, P_{T_{2}}$, and $P_{T_{3}}$ being the three vertices of triangle $T$. The associated discrete analogue of the ADMM algorithm described in section 3.2 reads as follows: Let $\left(\mathbf{u}_{h}^{0}, \boldsymbol{\lambda}_{h}^{0}\right) \in \mathbf{V}_{\mathbf{g}, h} \times \mathbf{Q}_{h}$ be given. Then, for $n \geq 1,\left(\mathbf{u}_{h}^{n-1}, \boldsymbol{\lambda}_{h}^{n-1}\right)$ being known, we compute $\mathbf{p}_{h}^{n}, \mathbf{u}_{h}^{n}$, and $\boldsymbol{\lambda}_{h}^{n}$ as follows: 
1. To obtain $\mathbf{p}_{h}^{n}$, solve

$$
\mathbf{p}_{h}^{n}=\arg \min _{\mathbf{q}_{h} \in \mathbf{Q}_{f h}}\left[\frac{r}{2}\left|\left\|\mathbf{q}_{h}\right\|\right|_{0 h}^{2}-\left(\left(\mathbf{X}_{h}^{n-1}, \mathbf{q}_{h}\right)\right)_{0 h}\right],
$$

where $\mathbf{X}_{h}^{n-1}:=r \nabla \mathbf{u}_{h}^{n-1}+\boldsymbol{\lambda}_{h}^{n-1}\left(\in \mathbf{Q}_{h}\right)$. Since no derivative of $\mathbf{q}$ is involved, problem (34) can be solved trianglewise using the method we employed in section 3.3 to solve problem (18) (of course, now $f(\mathbf{x})$ has to be replaced by $\left.\bar{f}_{T}\right)$.

2. To obtain $\mathbf{u}_{h}^{n}$, solve

$$
\mathbf{u}_{h}^{n}=\arg \min _{\mathbf{v}_{h} \in \mathbf{V}_{\mathbf{g}, h}}\left[\frac{\delta}{2}\|\boldsymbol{\theta}\|_{0 h}^{2}+\frac{1+r}{2}\|\nabla \mathbf{v}\|_{0 h}^{2}-\left(\left(\mathbf{Y}_{h}^{n}, \nabla \mathbf{v}_{h}\right)\right)_{0 h}\right],
$$

where $\mathbf{Y}_{h}^{n}:=\mathbf{I}_{d}+r \mathbf{p}_{h}^{n}-\boldsymbol{\lambda}_{h}^{n-1}\left(\in \mathbf{Q}_{h}\right)$, and where $\boldsymbol{\theta}$ (the discrete Laplacian of $-\mathbf{v}$ ) is uniquely obtained from $\mathbf{v}$, via the solution of

$$
\left\{\begin{array}{l}
\theta \in \mathbf{V}_{0 h}, \\
(\theta, \varphi)_{0 h}=((\nabla \mathbf{v}, \nabla \varphi))_{0 h}, \forall \boldsymbol{\varphi} \in \mathbf{V}_{0 h} .
\end{array}\right.
$$

The Euler-Lagrange equation (a system in fact) associated with the minimization problem (35), (36) reads as

$$
\left\{\begin{array}{l}
\left(\mathbf{u}_{h}^{n}, \boldsymbol{\omega}_{h}^{n}\right) \in \mathbf{V}_{\mathbf{g}, h} \times \mathbf{V}_{0 h}, \\
\delta\left(\boldsymbol{\omega}_{h}^{n}, \boldsymbol{\theta}\right)_{0 h}+(1+r)\left(\left(\nabla \mathbf{u}_{h}^{n}, \nabla \mathbf{v}\right)\right)_{0 h}=\left(\left(\mathbf{Y}_{h}^{n}, \nabla \mathbf{v}\right)\right)_{0 h}, \\
\forall(\mathbf{v}, \boldsymbol{\theta}) \in \mathbf{V}_{0 h} \times \mathbf{V}_{0 h}, \\
\left(\boldsymbol{\omega}_{h}^{n}, \boldsymbol{\varphi}\right)_{0 h}=\left(\left(\nabla \mathbf{u}_{h}^{n}, \nabla \boldsymbol{\varphi}\right)\right)_{0 h}, \forall \boldsymbol{\varphi} \in \mathbf{V}_{0 h} .
\end{array}\right.
$$

The variational system (37) is nothing but the mixed variational formulation of a discrete biharmonic problem. Similar problems have been encountered in, e.g., $[9,27]$ when solving the two-dimensional Monge-Ampère equation by least-squares methods operating in subsets of $H^{2}(\Omega)$. Solving problem (37) is quite simple since relation (36) implies the equivalence between (37) and the following system of discrete second order linear elliptic problems (a discrete analogue of system (32), (33)):

$$
\begin{aligned}
& \left\{\begin{array}{l}
\boldsymbol{\omega}_{h}^{n} \in \mathbf{V}_{0 h}, \\
(1+r)\left(\omega_{h}^{n}, \mathbf{v}\right)_{0 h}+\delta\left(\left(\nabla \boldsymbol{\omega}_{h}^{n}, \nabla \mathbf{v}\right)\right)_{0 h}=\left(\left(\mathbf{Y}^{n}, \nabla \mathbf{v}\right)\right)_{0 h} \\
\forall \mathbf{v} \in \mathbf{V}_{0 h},
\end{array}\right. \\
& \left\{\begin{array}{l}
\mathbf{u}_{n}^{n} \in \mathbf{V}_{g h}, \\
\left(\left(\nabla \mathbf{u}_{h}^{n}, \nabla \mathbf{v}\right)\right)_{0 h}=\left(\boldsymbol{\omega}_{h}^{n}, \mathbf{v}\right)_{0 h}, \\
\forall \mathbf{v} \in \mathbf{V}_{0 h} .
\end{array}\right.
\end{aligned}
$$

Solving numerically problems like (38) and (39) is routine nowadays. 
3. Finally we update the multiplier $\boldsymbol{\lambda}_{h}^{n-1}$ via

$$
\boldsymbol{\lambda}_{h}^{n}=\boldsymbol{\lambda}_{h}^{n-1}+r\left(\nabla \mathbf{u}_{h}^{n}-\mathbf{p}_{h}^{n}\right)
$$

The convergence of the proposed algorithm is discussed in sections 5 and 6 from a numerical viewpoint, based on the evidence provided by numerical experiments. The convergence of Uzawa-type algorithms such as the one proposed here have been discussed in [25]. However, due to the nonconvexity of the minimization problem under consideration, a mathematical proof of the convergence of the ADMM algorithm we employ still seems to be out of reach. Individually, the algebraic problems (34) are local problems that are identical at both the continuous and discrete levels. Convergence is ensured by the underlying properties of Newton methods. The convergence of finite element methods for biharmonic problems such as (37) is discussed, e.g., in [12].

Remark 4.1. As well-documented (see, e.g., [30] for further details and references), a critical issue with ADMM-type algorithms is the proper choice of the augmentation parameters, particularly for nonconvex problems. Least-squares/relaxation methods are an existing alternative to ADMM algorithms (see, e.g., [9] for the application to the Monge-Ampère equation in two dimensions of space). Concerning the finite element approximation of the prescribed Jacobian equation, possible extensions are higher order finite element approximations and adaptive mesh refinement methods (to better track the possible singularities of the solution, see section 6).

\section{Numerical experiments I. Benchmarks and validations.}

5.1. Generalities. In order to validate the numerical methodology discussed in the preceding sections, we consider first test cases with known exact solutions. Then we investigate situations where solutions are unknown or do not exist, and study the behavior of our ADMM algorithm for those situations. In particular, if solutions do not exist, we are interested to know whether the algorithm converges, in some sense, to some generalized solution, as close variants of it do when applied to the solution of the Dirichlet problem for the Monge-Ampère equation $\operatorname{det}^{2} u=f$, when no classical solution exists, due to the incompatibility between $f$ and the boundary data (see [18] for details). The first numerical experiment is borrowed from [6] for validation purposes and convergence study. The initial guess is $\left\{\mathbf{u}^{0}, \boldsymbol{\lambda}^{0}\right\}=\{\mathbf{0}, \mathbf{0}\}$, unless specified otherwise.

Remark 5.1. The stopping rule used by default is the distance between successive iterates $\left\|\mathbf{u}^{k}-\mathbf{u}^{k+1}\right\|_{0 h}$. It provides the robustness required when there is no guarantee of a classical solution. Alternative stopping criteria have been used in what follows for test cases with an exact solution; typically one can consider the residual $\left|\operatorname{det} \nabla \mathbf{u}^{k}-f\right|$, or the difference with an exact solution $\| \mathbf{u}^{k}-\mathbf{u}_{\text {exact }}||_{0 h}$. Numerical results are similar.

5.2. The identity mapping solution. We consider the open unit disk

$$
\Omega=\left\{\mathbf{x} \in \mathbb{R}^{2},\|\mathbf{x}\|_{2}<1\right\}
$$

(with $\Gamma=\partial \Omega$ ) and take as exact solution the vector-valued function $\mathbf{u}$ verifying $\mathbf{u}(\mathbf{x})=\mathbf{x}$ in $\Omega$ (that is, $\mathbf{u}$ is the identity mapping). The related (JAC) problem reads as follows: find $\mathbf{u}: \Omega \rightarrow \mathbb{R}^{2}$ satisfying

$$
\begin{cases}\operatorname{det} \nabla \mathbf{u}=1 & \text { in } \Omega, \\ \mathbf{u}(\mathbf{x})=\mathbf{x} & \text { a.e. } \mathbf{x} \text { on } \Gamma .\end{cases}
$$


From the introduction, we know that problem (40) has solutions other than the identity mapping. The set of numerical parameters is given by $r=10^{-6}$ and $\delta=10^{-6}$. The outer loop iteration stops if the tolerance between successive iterates $\mathbf{u}^{k}$ and $\mathbf{u}^{k+1}$ satisfies $\left\|\mathbf{u}^{k}-\mathbf{u}^{k+1}\right\|_{0 h}<\varepsilon=10^{-8}$. When computing $\mathbf{p}_{h}^{n}$ (via the solution of problem (34)), we took $10^{-5}$ as tolerance for Newton's method solving the associated discrete analogues of problem (24) and limited ourselves to 100 the number of Newton's iterations (a number which was never reached). The mesh is an unstructured Delaunay triangulation of $\Omega$ (see Figure 1). The algorithm we employed (namely the discrete analogue, described in section 3, of the ADMM algorithm (18), (21), (23)) was always converging in fewer than 20 outer iterations (between 17 and 19 iterations, depending of the mesh size).
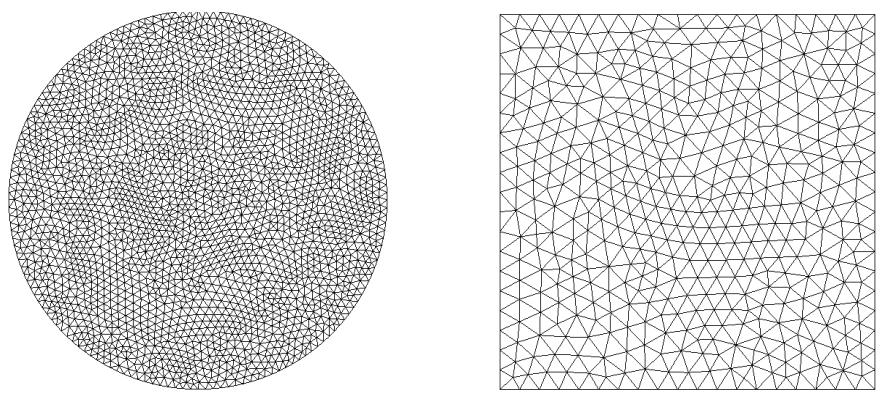

FIG. 1. Typical finite element triangulations used for the discretization of the unit disk and the unit square.

Figure 2 visualizes the solution on one particular mesh (with $h \simeq 0.0161$ ). The most natural solution $\mathbf{u}(\mathbf{x})=\mathbf{x}$ is correctly approximated, and the radial invariance is appropriately tracked even though the mesh does not guarantee such a symmetry. The determinant of $\mathbf{p}_{h}$ is exactly equal to one on each element (by construction), while the determinant of $\nabla \mathbf{u}_{h}$ is nearly everywhere equal to one (between 0.999998 and 1.000009), suggesting that the constraint $\nabla \mathbf{u}_{h}=\mathbf{p}_{h}$ is accurately satisfied (indeed our computations showed that $\left\|\left.\left|\nabla \mathbf{u}_{h}-\mathbf{p}_{h}\right|\right|_{0 h} /|| \mathbf{p}_{h} \mid\right\|_{0 h}=2.42 \cdot 10^{-4}$ in the particular case visualized in Figure 2).
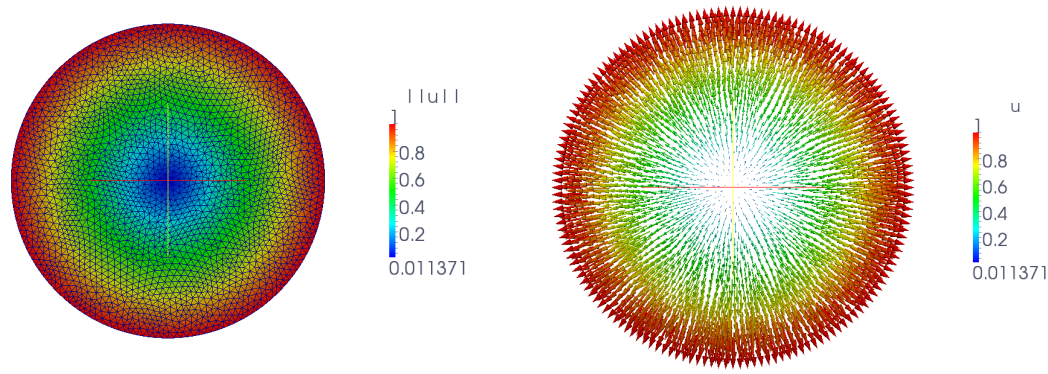

FIG. 2. The identity mapping on the unit disk. Visualization of the approximate solution obtained with the augmented Lagrangian approach after 19 iterations ( $h \simeq 0.0161$ ). Left: norm $\left\|\mathbf{u}_{h}\right\|_{2}$; Right: vector field $\mathbf{u}_{h}$;

The numerical results are similar when considering the unit square $\Omega=(0,1)^{2}$. 
Figure 3 visualizes the numerical results on the unit square for an unstructured mesh (similar to the one illustrated in Figure 1). Note that the results are actually similar when using structured meshes and thus independent of the type of mesh used.

Figure 4 illustrates the convergence of the error between the numerical solution $\mathbf{u}_{h}$ and the exact solution $\mathbf{u}(\mathbf{x})=\mathbf{x}$ for the unit disk and the unit square (with both structured and unstructured types of meshes). All configurations lead to the convergence with error approximation order $\mathcal{O}(h)$. The number of outer iterations of the algorithm is quite stable with respect to the mesh size, but depends on the test case considered.
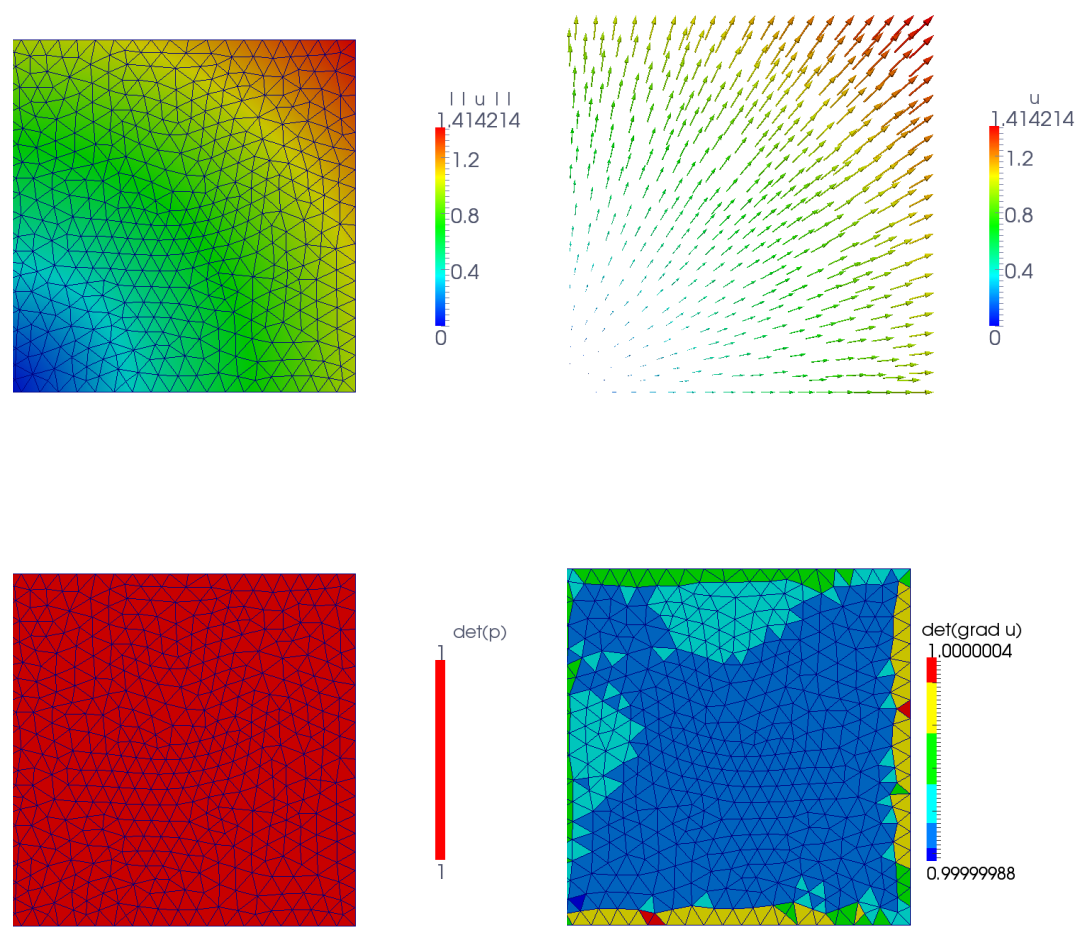

FIG. 3. The identity mapping on the unit square. Visualization of the approximate solution on the unit square obtained with the augmented Lagrangian approach after 24 iterations $(h \simeq 0.0220)$. Top left: norm $\left\|\mathbf{u}_{h}\right\|_{2}$; Top right: vector field $\mathbf{u}_{h} ;$ Bottom left: Determinant $\operatorname{det} \mathbf{p}_{h}$; Bottom right: Determinant $\operatorname{det} \nabla \mathbf{u}_{h}$.

Remark 5.2. Actually, for all test cases presented in this benchmark section, we could have taken $\delta=0$, meaning that the regularization term is not necessary when the test problem has a smooth solution.

6. Numerical experiments II. Extensions. During the second part of our numerical experiments, we consider more stringent (JAC) test problems in order to investigate the capabilities of our ADMM-based methodology. These test problems include (i) perturbations of problems with known exact solutions, (ii) problems with discontinuous data $f$ (including Dirac measures), and even (iii) problems where the nonexistence of solutions is known a priori since the compatibility condition (4) is not 


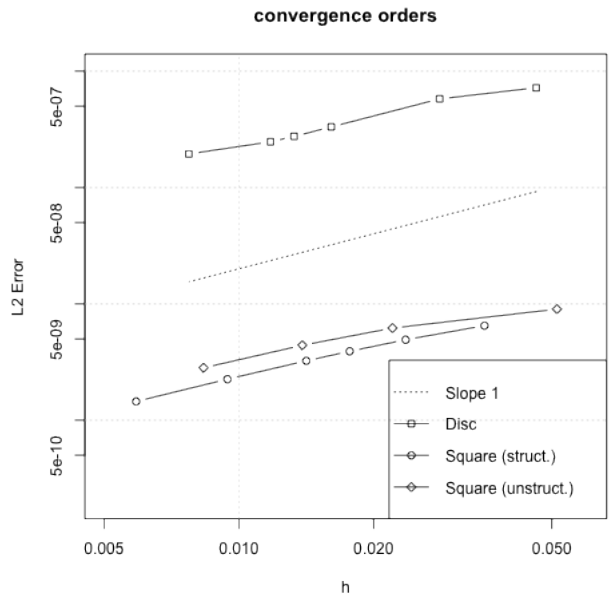

FIG. 4. The identity mapping for the unit disk and the unit square. Convergence of the $L^{2}$ approximation error $\left\|\mathbf{u}_{h}-\mathbf{u}\right\|_{0 h}$ for the unit disk (with unstructured mesh) and the unit square (with structured and unstructured meshes).

satisfied. In this section, the number of outer iterations for the augmented Lagrangian algorithm, typically of the order of 100 (unless otherwise specified), is chosen to be large enough to guarantee that the algorithm reaches a stationary state. Convergence can be reached faster in some cases.

6.1. A perturbation of the identity mapping. Let us consider again the unit square $\Omega=(0,1)^{2}$ (with coordinates denoted by $x_{1}$ and $x_{2}$ ). The (JAC) problem we consider now reads as follows: find $\mathbf{u}: \Omega \rightarrow \mathbb{R}^{2}$ satisfying

$$
\left\{\begin{array}{rlll}
\operatorname{det} \nabla \mathbf{u} & = & f & \text { in } \Omega \\
\mathbf{u}(\mathbf{x}) & = & \mathbf{x} & \text { on } \Gamma
\end{array}\right.
$$

with

$$
\begin{aligned}
f= & 1+\alpha \pi\left[\cos \left(\pi x_{1}\right) \sin \left(2 \pi x_{2}\right)+\sin \left(2 \pi x_{1}\right) \cos \left(\pi x_{2}\right)\right] \\
& +\alpha^{2} \pi^{2}\left[\cos \left(\pi x_{1}\right) \cos \left(\pi x_{2}\right) \sin \left(2 \pi x_{1}\right) \sin \left(2 \pi x_{2}\right)\right. \\
& \left.-4 \sin \left(\pi x_{1}\right) \sin \left(\pi x_{2}\right) \cos \left(2 \pi x_{1}\right) \cos \left(2 \pi x_{2}\right)\right]
\end{aligned}
$$

and

$$
\alpha= \pm \frac{1}{5 \pi}
$$

With this choice of the coefficient $\alpha$, it is easy to show that the right-hand side $f$ is positive on $\Omega$ and that an exact solution is the following perturbation of the identity mapping:

$$
\mathbf{u}\left(x_{1}, x_{2}\right)=\left(\begin{array}{c}
x_{1}+\alpha \sin \left(\pi x_{1}\right) \sin \left(2 \pi x_{2}\right) \\
x_{2}+\alpha \sin \left(2 \pi x_{1}\right) \sin \left(\pi x_{2}\right)
\end{array}\right) .
$$

We have visualized in Figure 5 the results obtained for a particular triangulation with $h=0.0354$ (the triangulation being a structured one where the square cells have been split into two triangles along the first diagonal). The constraint $\nabla \mathbf{u}=\mathbf{p}$ is satisfied up to the approximation errors, as illustrated by ||$\nabla \mathbf{u}_{h}-\mathbf{p}_{h}||_{0 h} /$ 
$\left\|\left|\mathbf{p}_{h}\right|\right\|_{0 h} \simeq 0.4151 \cdot 10^{-2}$. The convergence, for $h=0.0354$ given, is quantified by $\left\|\mathbf{u}_{h}^{50}-\mathbf{u}\right\|_{0 h} \simeq 0.449 \cdot 10^{-2}$. Figure 6 shows the order of convergence for the $L^{2}$-norm of the approximation error $\mathbf{u}-\mathbf{u}_{h}$; we observe again an $\mathcal{O}(h)$ (first order) convergence, significantly better results being obtained with structured mesh (probably due to a better fit between the shape of the exact solution on the domain and the preferred direction of the mesh).
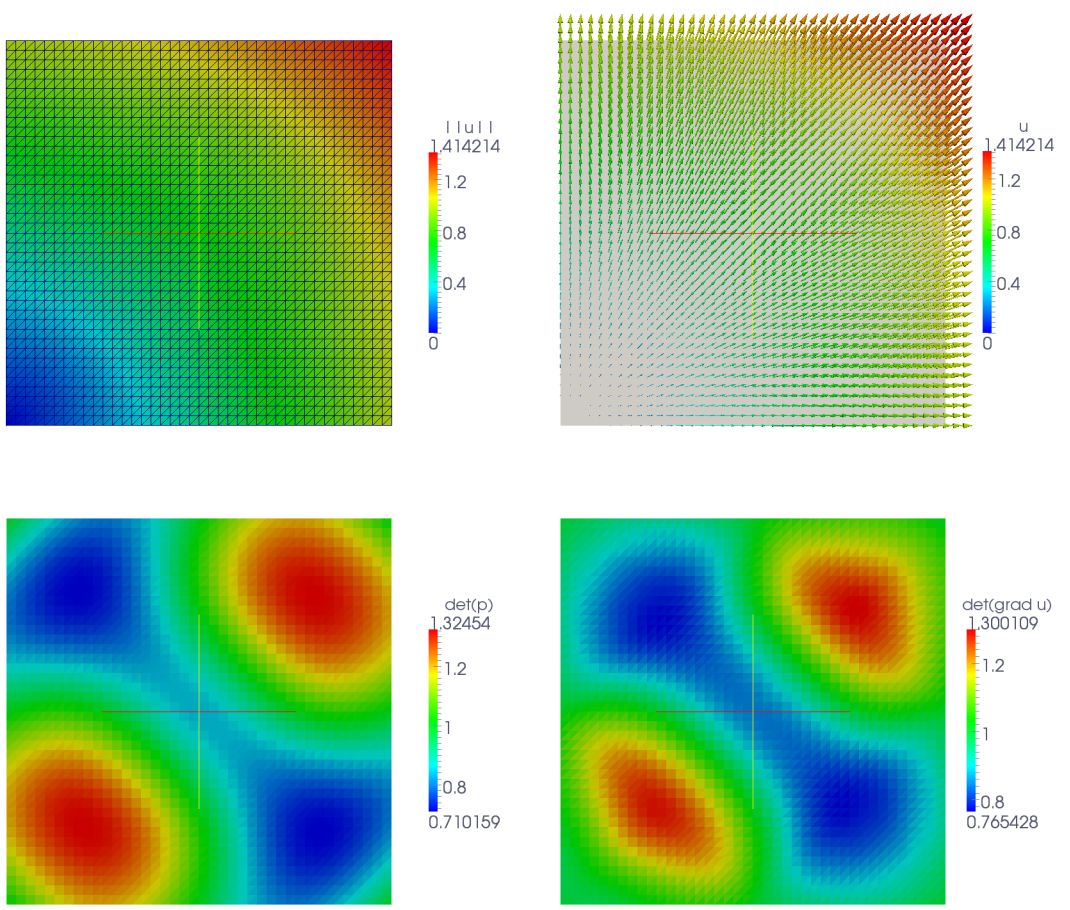

FIG. 5. Perturbation of the identity mapping on the unit square. Visualization of the approximate solution obtained with the augmented Lagrangian approach after 50 iterations $(h \simeq 0.0354)$. Top left: norm $\left\|\mathbf{u}_{h}\right\|_{2}$; Top right: vector field $\mathbf{u}_{h} ;$ Bottom left: Determinant $\operatorname{det} \mathbf{p}_{h}$; Bottom right: Determinant $\operatorname{det} \nabla \mathbf{u}_{h}$.

Increasing sufficiently the value of the parameter $\alpha$ leads to a right-hand side function $f$ that is no longer strictly positive. Actually, as soon as the parameter $\alpha$ makes the function $f$ nonpositive, the algorithm does not converge.

Remark 6.1. For nontrivial test cases, including the nonsmooth test cases presented later, we advocate taking $\delta \simeq(1+r) h^{2}$ to account for boundary effects on the solution of (31) (and to balance the main diagonals of the two matrices associated with (31)). Taking $\delta=0$ does not allow the algorithm to converge.

6.2. A test problem with noncompatible data. Let us consider the unit disk $\Omega=\left\{\mathbf{x} \in \mathbb{R}^{2},\|\mathbf{x}\|_{2}<1\right\}$ and the following (JAC) problem:

$$
\begin{cases}\operatorname{det} \nabla \mathbf{u}=4 & \text { in } \Omega \\ \mathbf{u}(\mathbf{x})=\mathbf{x} & \text { on } \Gamma\end{cases}
$$




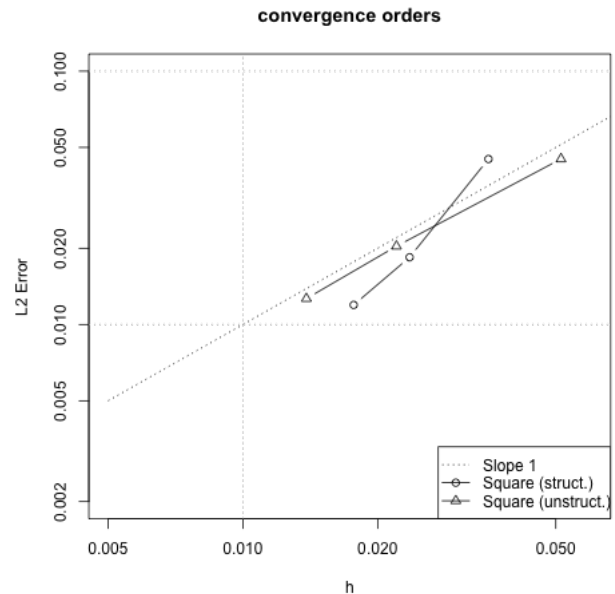

FIG. 6. Perturbation of the identity mapping on the unit square. Convergence of the approximation error $\left\|\mathbf{u}_{h}-\mathbf{u}\right\|_{0 h}$ (with structured or unstructured triangulations of the unit square).

This particular problem has no solution since it does not satisfy the compatibility condition (4); indeed we have here that

$$
\int_{\Omega} f d \mathbf{x}=4 \pi \neq \pi=\text { measure }(\Omega) .
$$

We apply the discrete ADMM algorithm discussed in section 4, with $r=10^{-1}$, $\delta=10^{-3}$, and $\left\{\mathbf{u}^{0}, \boldsymbol{\lambda}^{0}\right\}=\{\mathbf{0}, \mathbf{0}\}$, to approximate the solution of (42). We observe the following behavior:

1. We have $\mathbf{u}^{n}(\mathbf{x})=\mathbf{x}$ for all $\mathbf{x} \in \Omega$ and for all $n \geq 1$, which is the solution of $\operatorname{det} \nabla \mathbf{u}=1$ in $\Omega$ with $\mathbf{u}(\mathbf{x})=\mathbf{x}$ on $\Gamma$.

2. The determinant of $\mathbf{p}^{n}$ oscillates between the values 1 and 4 .

In this case, it is interesting to see that, from the incompatibility tracked by the augmented Lagrangian algorithm and the bad choice of the parameters $r$ and $\delta$, the algorithm does not converge. More precisely, the attractor for the solution $\mathbf{u}_{h}$ is given by $\mathbf{u}_{h}(\mathbf{x})=\mathbf{x}$ and remains constant throughout all the iterations (and satisfies the boundary conditions). On the other hand, the determinant of the solution $\mathbf{p}_{h}$ oscillates between the values 1 and 4; these oscillations show that the algorithm does not converge to any attractor point. The oscillations for $\mathbf{p}_{h}^{n}$ are natural, in the sense that they select an approximate solution that satisfies the differential equation (as planned) and an approximate solution that is influenced by the enforced boundary conditions.

In this case, the additional equality constraint $\nabla \mathbf{u}_{h}=\mathbf{p}_{h}$ cannot be satisfied and numerical oscillations are created. The results and behavior of the algorithm are similar if we take for $\Omega$ the unit square $(0,1)^{2}$.

In order to reduce, if not eliminate, the above oscillatory behavior, we increased the value of the augmentation parameter $r$, taking it equal to $r=100$ (with the corresponding $\delta$ equal to $\left.\delta=0.1 \simeq(1+r) h^{2}\right)$. With this value of $r$, the oscillations were significantly reduced and the following behavior was observed concerning the convergence of the sequence $\left\{\mathbf{u}_{h}^{n}, \mathbf{p}_{h}^{n}\right\}_{n}$ generated by the ADMM algorithm: (i) The sequence $\left\{\mathbf{p}_{h}^{n}\right\}_{n}$ verifies (by construction) $\operatorname{det} \mathbf{p}_{h}^{n}=4$ for all $n \geq 1$ and converges to a 
limit $\mathbf{p}_{h}$. (ii) $\mathbf{u}_{h}^{n}(\mathbf{x})=\mathbf{x}$ for all $\mathbf{x} \in \Omega$ and for all $n \geq 1$. (iii) The convergence is still oscillatory, but the oscillations are of smaller amplitudes.

6.3. Test problems with some radial symmetry. Assuming that $\Omega$ is still the unit disk $\Omega=\left\{\mathbf{x} \in \mathbb{R}^{2},\|\mathbf{x}\|_{2}<1\right\}$, we consider the following (JAC) problem, where $f$ is radial symmetric, namely

$$
\begin{cases}\operatorname{det} \nabla \mathbf{u}(\mathbf{x})=f\left(\|\mathbf{x}\|_{2}\right) & \text { in } \Omega, \\ \mathbf{u}(\mathbf{x})=\mathbf{g}(\mathbf{x}) & \text { on } \Gamma,\end{cases}
$$

where

$$
\mathbf{g}(\mathbf{x})=\sqrt{2}\left(\begin{array}{c}
\frac{1}{2}\left(x_{1}^{2}-x_{2}^{2}\right) \\
x_{1} x_{2}
\end{array}\right)
$$

Assume that

$$
f(\rho)=2 \rho^{2}
$$

we verify that, in this particular case, an exact solution of the determinant equation is given by

$$
\mathbf{u}(\mathbf{x})=\sqrt{2}\left(\begin{array}{c}
\frac{1}{2}\left(x_{1}^{2}-x_{2}^{2}\right) \\
x_{1} x_{2}
\end{array}\right),
$$

which is not a radial function. The uniqueness is a priori not guaranteed. The solution $\mathbf{u}$ of problem (43) is accurately approximated since, after 100 outer iterations, with $r=10^{-4}$ and $\delta=10^{-6} \simeq(1+r) h^{2}$, we have $\left\|\mathbf{u}_{h}^{100}-\mathbf{u}\right\|_{0 h} \simeq 7.74 \cdot 10^{-4}$ (while $\left.\| \mathbf{u}_{h}^{100}-\left.\mathbf{u}_{h}^{99}\right|_{0 h} \simeq 3.0 \cdot 10^{-6}\right)$. The solution is not radial, but its Euclidean norm is a radial function (since $\|\mathbf{u}\|_{2}^{2}=0.5 x_{1}^{4}+0.5 x_{2}^{4}+x_{+}^{2} x_{2}^{2}=0.5\left(x_{1}^{2}+x_{2}^{2}\right)^{2}$ ), a property well satisfied in Figure 7(right). Note that this approximate solution is actually obtained after five outer iterations and remains stationary without oscillations afterwards.
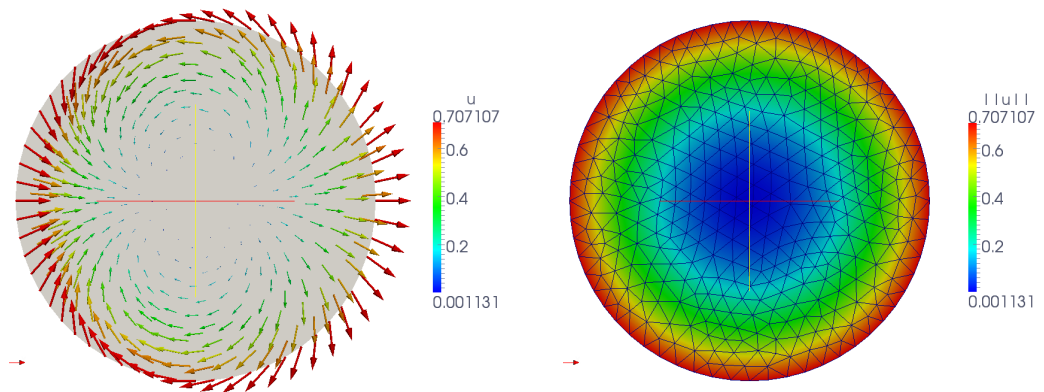

FIG. 7. Test problem with radial data. Visualization of the approximate solution on the unit disk obtained with the augmented Lagrangian approach after 100 iterations $\left(h \simeq 0.0461, r=10^{-4}\right.$, $\left.\delta=10^{-6}\right)$. Left: vector field $\mathbf{u}_{h}$. Right: norm $\left\|\mathbf{u}_{h}\right\|_{2}$.

When the value of the parameter $r$ is increased to $r=10$ (or anything larger), the parameter $\delta$ is set to $\delta=10^{-2} \simeq(1+r) h^{2}$, and the ADMM algorithm bifurcates to find an approximation of another branch of solutions. This second solution satisfies the boundary conditions, but its Euclidean norm is no longer radial. This illustrates the nonuniqueness of the solution to the (JAC) problem for this particular set of data and the capability of the algorithm to capture multiple solutions if properly monitored. 
This bifurcation effect is classical for augmented Lagrangian algorithms and has been observed already in, e.g., [8]. Numerical results are visualized in Figure 8.
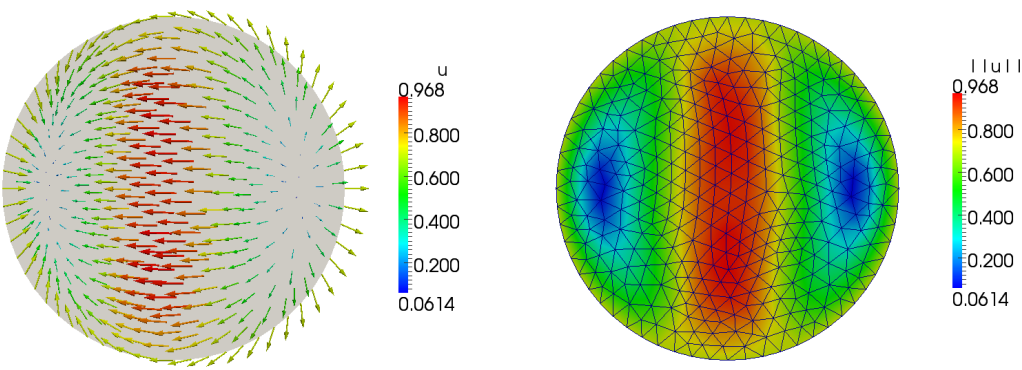

FIG. 8. Test problem with radial data. Visualization of the approximate solution on the unit disk obtained with the augmented Lagrangian approach after 100 iterations $(h \simeq 0.0461, r=10$, $\delta=0.01)$. Left: vector field $\mathbf{u}_{h}$. Right: norm $\left\|\mathbf{u}_{h}\right\|_{2}$.

When considering $\mathbf{g}(\mathbf{x})=\mathbf{x}$ in (43), an exact solution of the (JAC) problem is

$$
\mathbf{u}(\mathbf{x})=\|\mathbf{x}\|_{2}\left(\begin{array}{l}
x_{1} \\
x_{2}
\end{array}\right),
$$

which is a radial function. With $r=10$ and $\delta=10^{-2} \simeq(1+r) h^{2}$, the ADMM algorithm presented in section 4 approximates the above exact solution after about 100 iterations with $\left\|\mathbf{u}_{h}^{100}-\mathbf{u}\right\|_{0 h} \simeq 9.99 \cdot 10^{-3}$. Results are illustrated in Figure 9 .
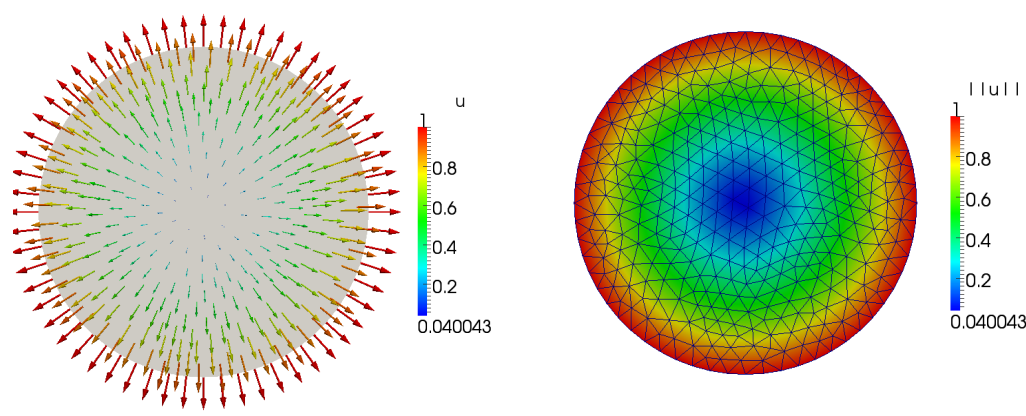

FIG. 9. Test problem with radial data and $\mathbf{g}(\mathbf{x})=\mathbf{x}$. Visualization of the approximate solution on the unit disk obtained with the augmented Lagrangian approach after 100 iterations $(h \simeq 0.0461$, $\left.r=10, \delta=10^{-2}\right)$. Left: vector field $\mathbf{u}_{h}$. Right: norm $\left\|\mathbf{u}_{h}\right\|_{2}$.

6.4. Test problems for some nonconvex domains with re-entrant corners. The solution of (JAC) for nonconvex domains has not been addressed in the literature. Let us consider first the pie-shaped domain obtained by removing from the 
unit disk a piece of angular size $2 \alpha$ (as shown in Figure 10, where $\alpha=\pi / 4$ degrees):

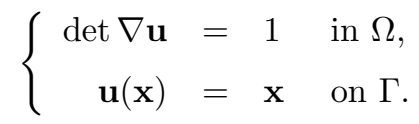

An exact solution of problem (44) is given by

$$
\mathbf{u}\left(x_{1}, x_{2}\right)=\left(\begin{array}{l}
x_{1} \\
x_{2}
\end{array}\right) .
$$

Taking $r=10^{4}$ and $\delta=1$, we initialize the ADMM algorithm of section 4 with $\boldsymbol{\lambda}^{0}=0$ and $\mathbf{u}^{0}(\mathbf{x})=\mathbf{0}$ in $\Omega$, and $\mathbf{u}^{0}(\mathbf{x})=\mathbf{x}$ on $\Gamma$. In Figure 10 we have visualized the results obtained after 100 iterations, using a triangulation refined in the neighborhood of the re-entrant corner (we observed, not surprisingly, that such a refinement enhances the convergence of our iterative method). The exact solution is accurately recovered (since $\left.\left\|\mathbf{u}_{h}^{100}-\mathbf{u}\right\|_{0 h} \simeq 0.323 \cdot 10^{-3}\right)$.

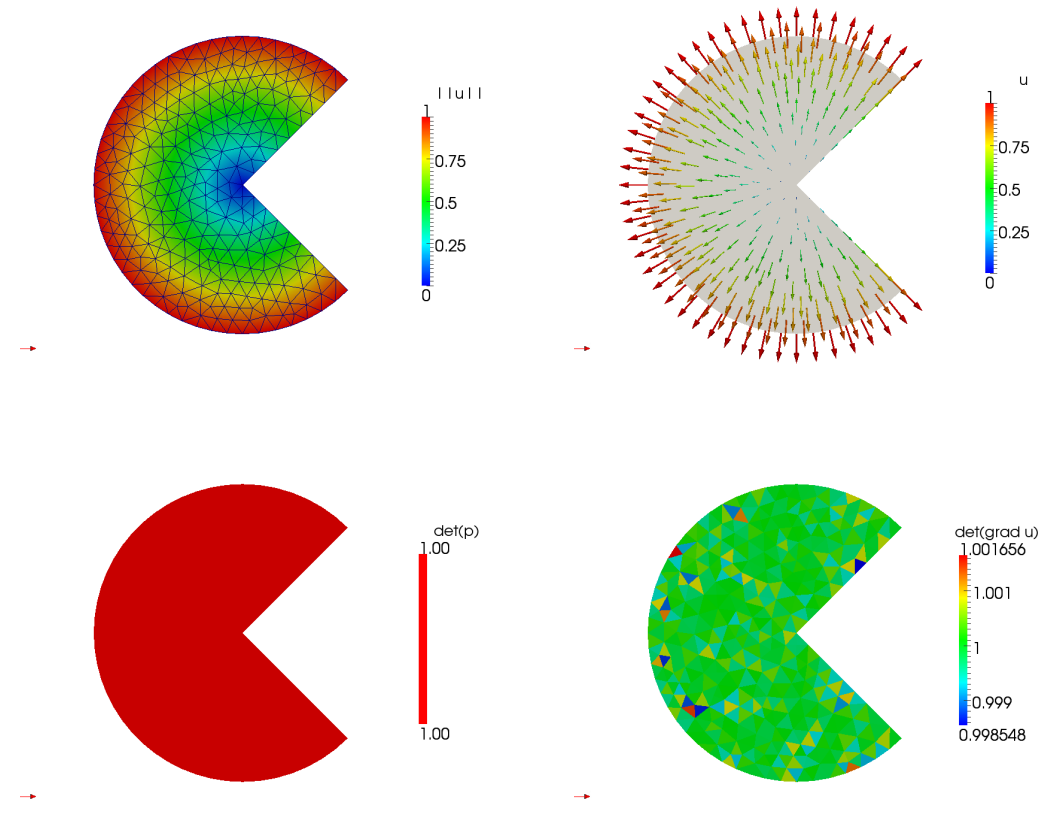

FIG. 10. Test problem for a pie-shaped domain (with $\alpha=\pi / 4$ degrees) and $f=1$. Visualization of the approximate solution obtained with the augmented Lagrangian approach after 200 iterations ( $h \simeq 0.0263$ ). Top left: norm $\left\|\mathbf{u}_{h}\right\|_{2}$; Top right: vector field $\mathbf{u}_{h} ;$ Bottom left: determinant $\operatorname{det} \mathbf{p}_{h}$; Bottom right: determinant $\operatorname{det} \nabla \mathbf{u}_{h}$.

Then we replace $f=1$ by $f(\rho)=2 \rho^{2}$ in (44), taking $r=10, \delta=10^{-3}, \lambda^{0}=0$, and $\mathbf{u}^{0}(\mathbf{x})=\mathbf{x}$ in $\Omega$, and $\mathbf{u}^{0}(\mathbf{x})=\mathbf{0}$ on $\Gamma$. In Figure 11 we have visualized the results obtained after 300 iterations on the same triangulation. Results are extremely consistent with those obtained on the unit disk in Figure 7.

We consider next the same pie-shaped domain with $\alpha=\pi / 100$ degrees, as illustrated in Figure 12. This (JAC) problem is defined as follows: 

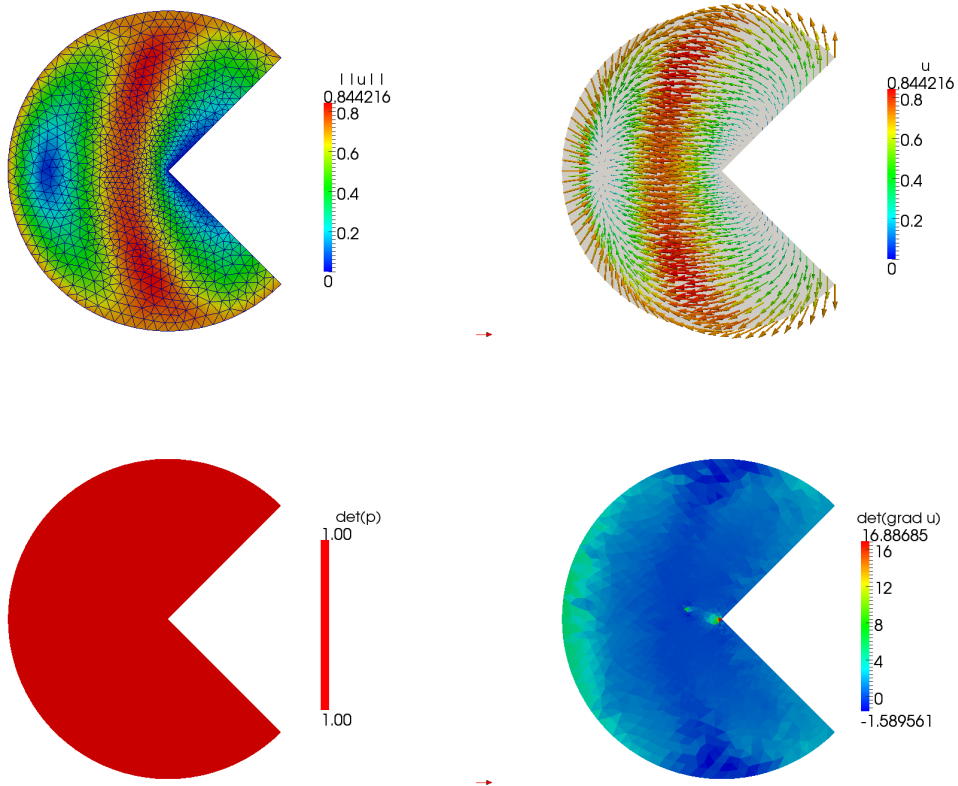

Fig. 11. Test problem for a pie-shaped domain (with $\alpha=\pi / 4$ degrees) and $f(\rho)=2 \rho^{2}$. Visualization of the approximate solution obtained with the augmented Lagrangian approach after 200 iterations $(h \simeq 0.0263)$. Top left: norm $\left\|\mathbf{u}_{h}\right\|_{2}$; Top right: vector field $\mathbf{u}_{h}$; Bottom left: determinant $\operatorname{det} \mathbf{p}_{h} ;$ Bottom right: determinant $\operatorname{det} \nabla \mathbf{u}_{h}$.

$$
\left\{\begin{array}{rll}
\operatorname{det} \nabla \mathbf{u} & =1 & \text { in } \Omega, \\
\mathbf{u}(\mathbf{x}) & =\mathbf{x} & \text { on } \Gamma .
\end{array}\right.
$$

Again, the triangulation we employed was refined around the re-entrant corner. Taking $r=10^{5}, \delta=1$, we initialize the ADMM algorithm of section 4 with $\boldsymbol{\lambda}^{0}=\mathbf{0}$ and $\mathbf{u}^{0}=\mathbf{0}$, leading to the results reported in Figure 12, obtained after 100 iterations. The exact solution has been correctly recovered (since $\left\|\mathbf{u}_{h}^{100}-\mathbf{u}\right\|_{0 h}=0.371 \cdot 10^{-3}$ when $h=0.0480$ ); the convergence is more oscillatory than when the re-entrant angle is larger (which was expected).

6.5. Two test problems with nonsmooth right-hand sides. Let us consider now test problems involving discontinuous data $f$. The lack of regularity of the following data is expected to lead to low regularity solutions to problem (2), if any (see, e.g., [17]). With $\Omega$ being the unit $\operatorname{disk} \Omega=\left\{\mathbf{x} \in \mathbb{R}^{2},\|\mathbf{x}\|_{2}<1\right\}$, we consider first the following problem:

$$
\begin{cases}\operatorname{det} \nabla \mathbf{u}=f & \text { in } \Omega \\ \mathbf{u}(\mathbf{x})=\mathbf{x} & \text { on } \Gamma\end{cases}
$$

with

$$
f(\mathbf{x})= \begin{cases}1.8 & \text { if } x_{1}>0 \\ 0.2 & \text { if } x_{1} \leq 0\end{cases}
$$



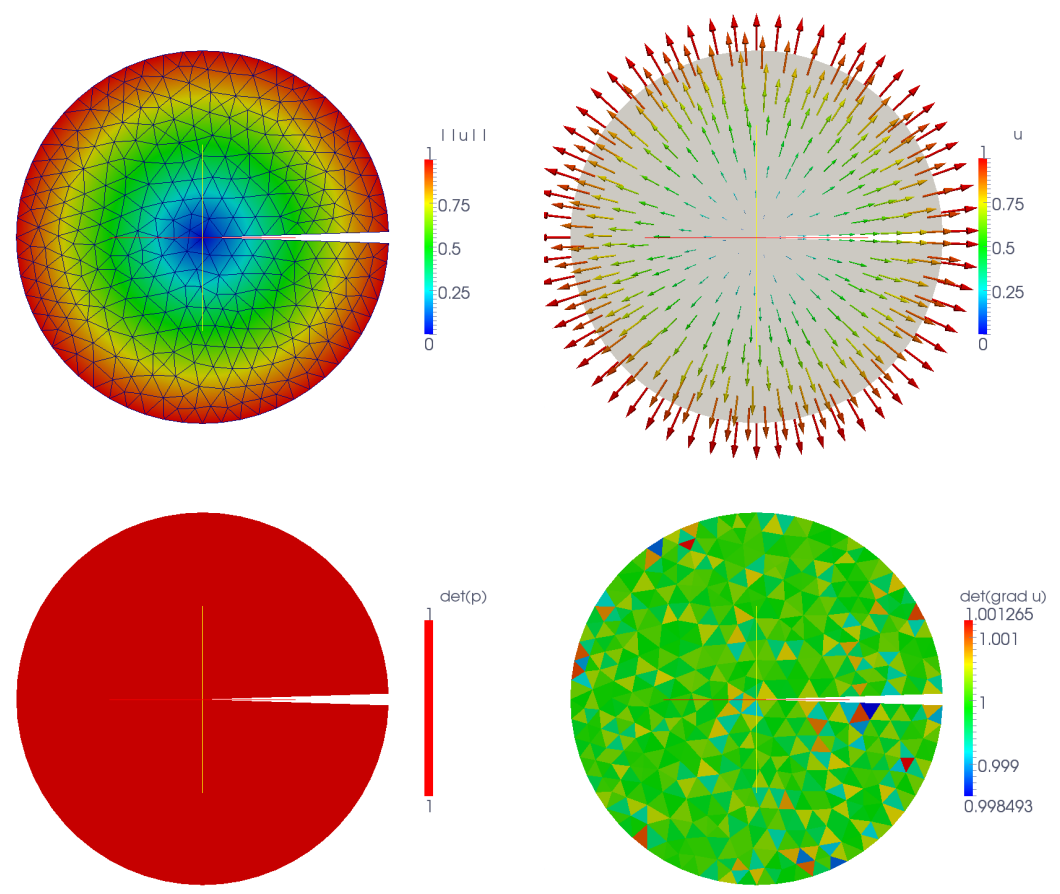

FIG. 12. Test problem for a pie-shaped domain (with $\alpha=\pi / 100$ degrees) and $f=1$. Visualization of the approximate solution obtained with the augmented Lagrangian approach after 100 iterations $(h \simeq 0.0480)$. Top left: norm $\left\|\mathbf{u}_{h}\right\|_{2} ;$ Top right: vector field $\mathbf{u}_{h} ;$ Bottom left: determinant $\operatorname{det} \mathbf{p}_{h} ;$ Bottom right: determinant $\operatorname{det} \nabla \mathbf{u}_{h}$.

We observe that $f$ is compatible in the sense that $\int_{\Omega} f d \mathbf{x}=\pi=$ measure $(\Omega)$. Starting from $\left\{\mathbf{u}^{0}, \boldsymbol{\lambda}^{0}\right\}=\{\mathbf{0}, \mathbf{0}\}$, and using $r=10$ and $\delta=0.0001$, the ADMM algorithm converges after 100 iterations to the solution $\mathbf{u}_{h}$, shown in Figure 13. We use a triangulation refined along the vertical axis in the neighborhood of the singularity of the right-hand side. The function $\operatorname{det}\left(\mathbf{p}_{h}\right)$ is equal to $f$ by construction (up to mesh effects), but significantly different from $\operatorname{det}\left(\nabla \mathbf{u}_{h}\right)$, since $\mathbf{u}_{h}$ is smoother due to the biharmonic regularization.

Remark 6.2. The effect of the parameter $\delta$ on the solution $\mathbf{u}_{h}$ is quantified as follows. Let us take $\delta=1,10^{-2}$ and $10^{-4}(r=10$ being fixed). Figure 14 shows $\left\|\mathbf{u}_{h}\right\|_{0 h}$ and $\operatorname{det} \nabla \mathbf{u}_{h}$ for these values of $\delta$ on a nonrefined mesh. We can observe that, when $\delta$ is too large, the solution and the determinant of its gradient are smoothed drastically. When $\delta$ decreases, we recover the sharpness of the solution. When $\delta$ becomes too small, the quality of the solution does not improve significantly, but the convergence of the iterative algorithm deteriorates.

The second test problem with a nonsmooth right-hand side that we consider is defined by

$$
\begin{cases}\operatorname{det} \nabla \mathbf{u}=f & \text { in } \Omega \\ \mathbf{u}(\mathbf{x})=\mathbf{x} & \text { on } \Gamma,\end{cases}
$$

where, once again, $\Omega$ is the unit disk $\Omega=\left\{\mathbf{x} \in \mathbb{R}^{2},\|\mathbf{x}\|_{2}<1\right\}$ and

$$
f(\mathbf{x})=\pi \delta_{(\mathbf{0})},
$$



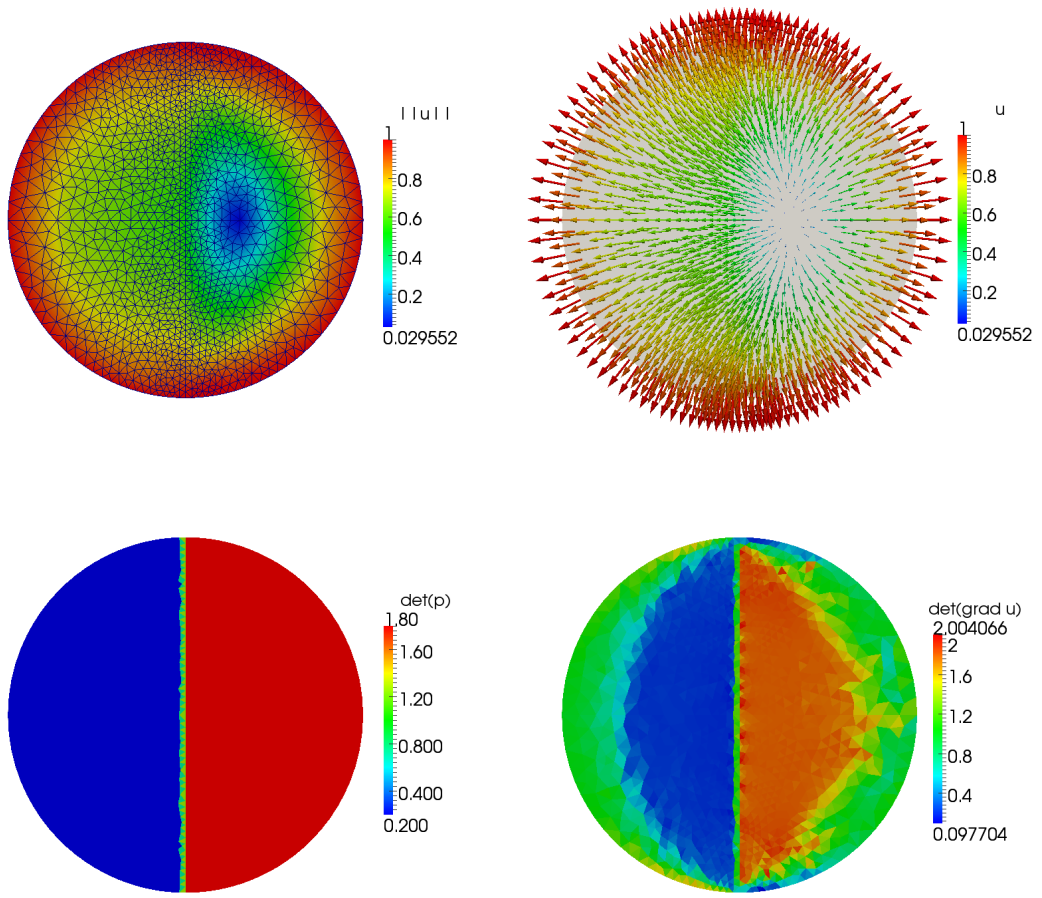

FIG. 13. Test problem with a discontinuous right-hand side (with $f$ given by (47)). Visualization of the approximate solution obtained with the augmented Lagrangian approach after 100 iterations ( $h \simeq 0.0461, r=10, \delta=0.01)$. Top left: norm $\left\|\mathbf{u}_{h}\right\|_{2} ;$ Top right: vector field $\mathbf{u}_{h} ;$ Bottom left: determinant $\operatorname{det} \mathbf{p}_{h} ;$ Bottom right: determinant $\operatorname{det} \nabla \mathbf{u}_{h}$.

$\delta_{(\mathbf{0})}$ being the Dirac measure at $\mathbf{0}=(0,0)$. An exact solution to problem (48) is given by

$$
\mathbf{u}_{0}(\mathbf{x})=\frac{\mathbf{x}}{\|\mathbf{x}\|_{2}} .
$$

The function $\mathbf{u}_{0} \notin H_{l o c}^{1}\left(\mathbb{R}^{2}\right)$, implying that problem (48) is not relevant (directly, at least) to the framework detailed in [17]. To overcome this difficulty, we proceed by regularization as in [9], replacing $f$ by $f_{\eta}$ defined by

$$
f_{\eta}(\mathbf{x})=\frac{\left(1+\eta^{2}\right) \eta^{2}}{\left(\eta^{2}+\|\mathbf{x}\|_{2}^{2}\right)^{2}}
$$

where $\eta$ is a positive parameter; indeed,

$$
\lim _{\eta \rightarrow 0} f_{\eta}=\pi \delta_{(\mathbf{0})}
$$

in the sense of distributions. The corresponding approximation of problem (48) reads as

$$
\begin{cases}\operatorname{det} \nabla \mathbf{u}_{\eta}=f_{\eta} & \text { in } \Omega, \\ \mathbf{u}(\mathbf{x})=\mathbf{x} & \text { on } \Gamma\end{cases}
$$



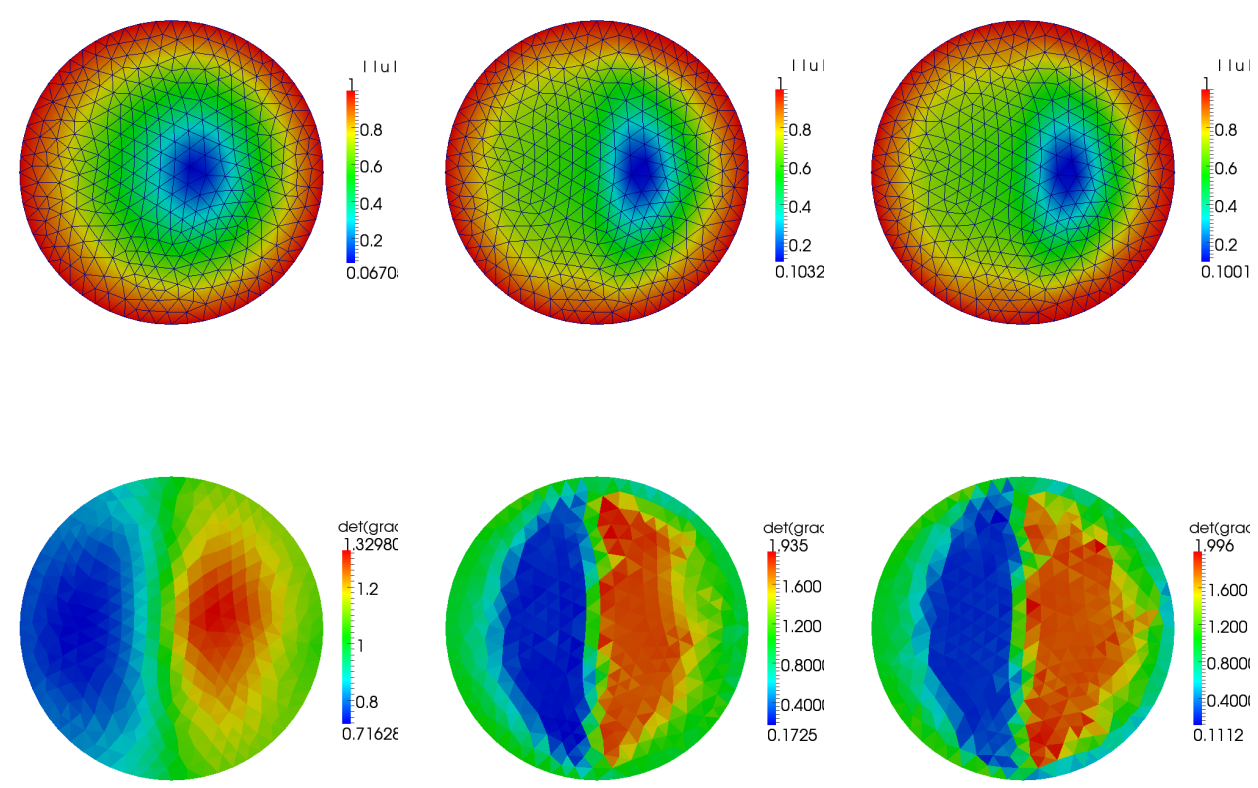

FIG. 14. Test problem with a discontinuous right-hand side. Visualization of the approximate solution obtained with the augmented Lagrangian approach after 100 iterations $(h \simeq 0.0461, r=$ $\left.10, f=f_{2}\right)$, on a triangulation not refined along the singularity. Top: norm $\left\|\mathbf{u}_{h}\right\|_{2} ;$ Bottom: determinant $\operatorname{det} \nabla \mathbf{u}_{h}$. Left: $\delta=1$, middle: $\delta=10^{-2} ;$ Right: $\delta=10^{-4}$.

and has $\mathbf{u}_{\eta}$ defined by

$$
\mathbf{u}_{\eta}(\mathbf{x})=\mathbf{x} \sqrt{\frac{1+\eta^{2}}{\eta^{2}+\|\mathbf{x}\|_{2}^{2}}}
$$

as an exact solution. We clearly have $\mathbf{u}_{\eta} \in C^{\infty}(\bar{\Omega})$ for all $\eta>0$ and can (relatively) easily show that

$$
\left\|\mathbf{u}_{\eta}-\mathbf{u}_{0}\right\|_{L^{2}(\Omega)}=\mathcal{O}(\eta) .
$$

For large values of $\eta$, the function $\mathbf{u}_{\eta}$ does not exhibit strong gradients in $\bar{\Omega}$ and, not surprisingly, the ADMM algorithm proves efficient and accurate. On the other hand, $\mathbf{u}_{\eta}$ develops, as expected, a singularity with strong gradients near $(0,0)$ as $\eta \rightarrow 0_{+}$ requiring global or local mesh refinement to resolve this singularity.

We have visualized in Figure 15 the computed solution obtained at the 100th iteration of the discrete ADMM algorithm discussed in section 4, taking $h=0.005, r=$ $10, \delta=0.01$, and $\eta=1 / 8$ and using $\left.\left(\mathbf{u}^{0}, \boldsymbol{\lambda}^{0}\right)\right)=(\mathbf{0}, \mathbf{0})$ as initializer. The exact solution of the regularized problem (defined by (52)) is approximated accurately, as shown in Table 1, the same conclusion holding for the other values of $\eta$, as shown also in Figure 16. Indeed, Figure 16 suggests $\left\|\mathbf{u}_{\eta, h}-\mathbf{u}_{\eta}\right\|_{L^{2}(\Omega)}=\mathcal{O}(h)$ approximately (and possibly better as $h$ decreases), implying $\left\|\mathbf{u}_{\eta, h}-\mathbf{u}_{0}\right\|_{L^{2}(\Omega)} \simeq \mathcal{O}(\eta+h)$.

Figures 15,17 , and 18 show also that for $\eta<<1$ and $h$ sufficiently small the properties $\left\|\mathbf{u}_{0}(\mathbf{x})\right\|_{2}=1$ and $\operatorname{det} \nabla \mathbf{u}_{0}(\mathbf{x})=0$ for all $\mathbf{x} \in \bar{\Omega} \backslash\{(0,0)\}$ are accurately 

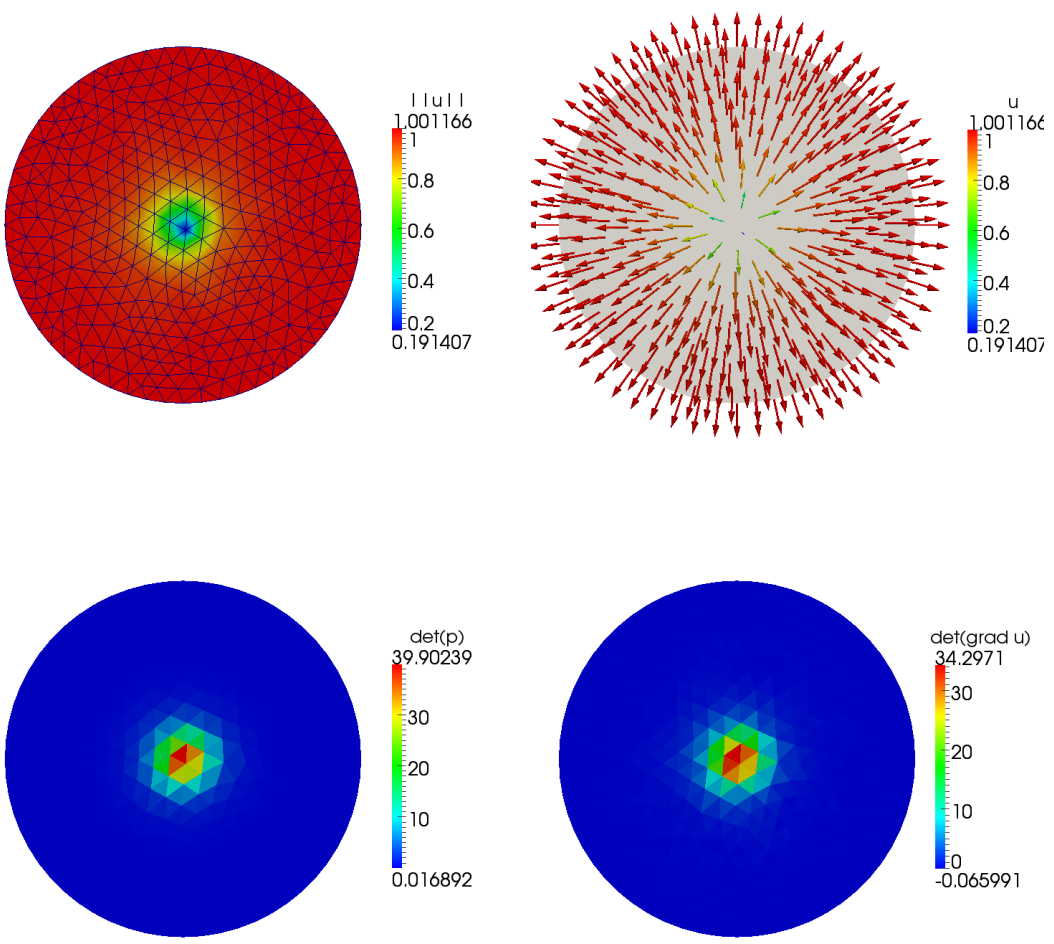

FIG. 15. Test problem with $f=\pi \delta_{(\mathbf{0})}$. Visualization of the approximate solution obtained by the augmented Lagrangian approach after 100 iterations $(h \simeq 0.005, r=10, \delta=0.01, \eta=1 / 8)$. Top left: norm $\left\|\mathbf{u}_{h}\right\|_{2}$; Top right: vector field $\mathbf{u}_{h}$; Bottom left: determinant $\operatorname{det} \mathbf{p}_{h} ;$ Bottom right: determinant $\operatorname{det} \nabla \mathbf{u}_{h}$.

satisfied, the lack of convergence due to the singularity at $(0,0)$ being localized in a small patch of triangles centered at $(0,0)$ and whose area converges to 0 as $(h, \eta) \rightarrow$ $(0,0)$, as particularly well reported in Figure 18.

Remark 6.3. When approximating $\operatorname{sgn} \xi$ by $\frac{\xi}{\sqrt{\xi^{2}+\eta^{2}}}$ the jump at $\xi=0$ is replaced by a continuous layer, centered at 0 , of thickness $\simeq 2 \eta$. A similar result holds when approximating $\mathbf{u}_{0}$ by $\mathbf{u}_{\eta}$, implying taking $h \ll \eta$ in order to resolve this transition layer.

To conclude this section, let us mention the following bifurcation phenomenon we observed when applying ADMM to the finite element approximation of problem (52). Suppose that $r, \delta$, and the triangulation $\mathcal{T}_{h}$ are fixed and that one decreases $\eta$ : below a critical value of $\eta$ (the bifurcation threshold here) ADMM stops converging to (an approximation of) $\mathbf{u}_{\eta}$ but converges instead to the function $\mathbf{x} \rightarrow \mathbf{x}$. Refining the mesh decreases the above bifurcation threshold. Similar bifurcation phenomena have been previously observed (see, e.g., [9]) when applying ADMM to the solution of nonconvex minimization problems. 
TABLE 1

Test problem with $f=\pi \delta_{(\mathbf{0})}$. Variations with $\eta$ and $h$ of the approximation error $\left\|\mathbf{u}_{\eta, h}-\mathbf{u}_{\eta}\right\|_{0, h}$. The function $\mathbf{u}_{\eta, h}$ has been obtained at the 100th iteration of the ADMM algorithm using $r=10$ and $\delta=0.01$.

\begin{tabular}{|c|c|c|c|}
\hline & \multicolumn{3}{|c|}{$\mid \mathbf{u}_{\eta, h}-\mathbf{u}_{\eta} \|_{0 h}$} \\
\hline$\eta$ & $h \simeq 0.0461$ & $h \simeq 0.0058$ & $h \simeq 0.0032$ \\
\hline 1 & $0.123378 \cdot 10^{-2}$ & $0.315747 \cdot 10^{-3}$ & $0.124853 \cdot 10^{-3}$ \\
$1 / 2$ & $0.458005 \cdot 10^{-2}$ & $0.138321 \cdot 10^{-2}$ & $0.277669 \cdot 10^{-3}$ \\
$1 / 4$ & $0.101486 \cdot 10^{-1}$ & $0.272539 \cdot 10^{-2}$ & $0.502128 \cdot 10^{-3}$ \\
$1 / 8$ & $0.225558 \cdot 10^{-1}$ & $0.568670 \cdot 10^{-2}$ & $0.933829 \cdot 10^{-3}$ \\
$1 / 16$ & $0.446432 \cdot 10^{-1}$ & $0.703255 \cdot 10^{-2}$ & $0.320679 \cdot 10^{-2}$ \\
$1 / 32$ & $0.877680 \cdot 10^{-1}$ & $0.425320 \cdot 10^{-1}$ & $0.733439 \cdot 10^{-1}$ \\
\hline
\end{tabular}

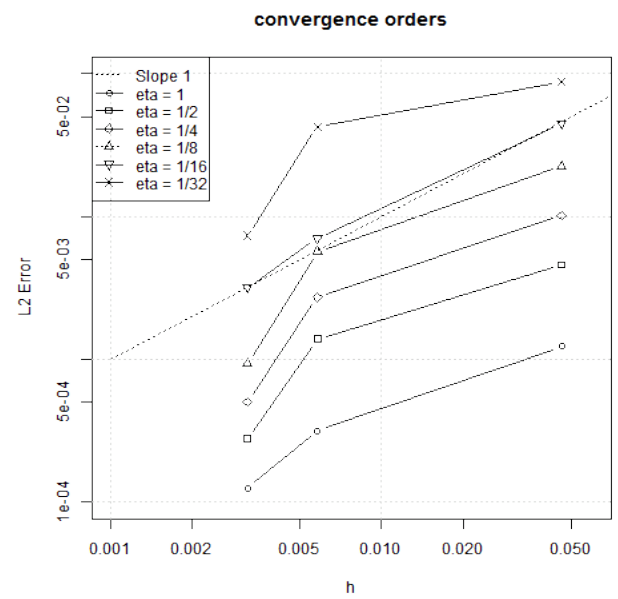

FIG. 16. Test problem with $f=\pi \delta_{(\mathbf{0})}$. Convergence of the discrete $L^{2}$ approximation error $\left\|\mathbf{u}_{h}-\mathbf{u}\right\|_{0 h}$ for various values of the regularization parameter $\eta$.

6.6. Test problems with a singularity on the boundary. Let us consider $\Omega=(0,1)^{2}$ and the problem

$$
\begin{cases}\operatorname{det} \nabla \mathbf{u}=0 & \text { in } \Omega, \\ \mathbf{u}(\mathbf{x})=\mathbf{x} /\|\mathbf{x}\|_{2} & \text { on } \Gamma \backslash\{(0,0)\},\end{cases}
$$

whose exact solution $\mathbf{u}(\mathbf{x})=\mathbf{x} /\|\mathbf{x}\|_{2}$ presents a singularity in the corner of $\Omega$. Note that this singularity is rather strong since the solution does not even exist in $(0,0)$. Therefore, we do not enforce Dirichlet boundary conditions in $(0,0)$, and we consider the regularized problem, where $f$ is given by (51), which admits the exact solution (53).

Taking $r=10^{-6}$ and $\delta=10^{-6}$, we initialize the ADMM algorithm of section 4 with $\boldsymbol{\lambda}^{0}=0$ and $\mathbf{u}^{0}(\mathbf{x})=\mathbf{0}$ in $\Omega$. The parameter $\eta$ is set to $10^{-2}$ (of the order of $h$ ), and the initial condition $\mathbf{u}^{0}(\mathbf{x})$ is given by (53) on $\Gamma$. In Figure 19 we have visualized the results obtained after 10 iterations (having reached stationarity of the algorithm), using a triangulation refined in the neighborhood of the corner hosting the singularity of the solution. One can observe that the singularity induces a dissipation in the wake of the point $(0,0)$. Nevertheless, the approximation of $\operatorname{det} \nabla \mathbf{u}_{h}$ is correct up to the 
$\eta=1$
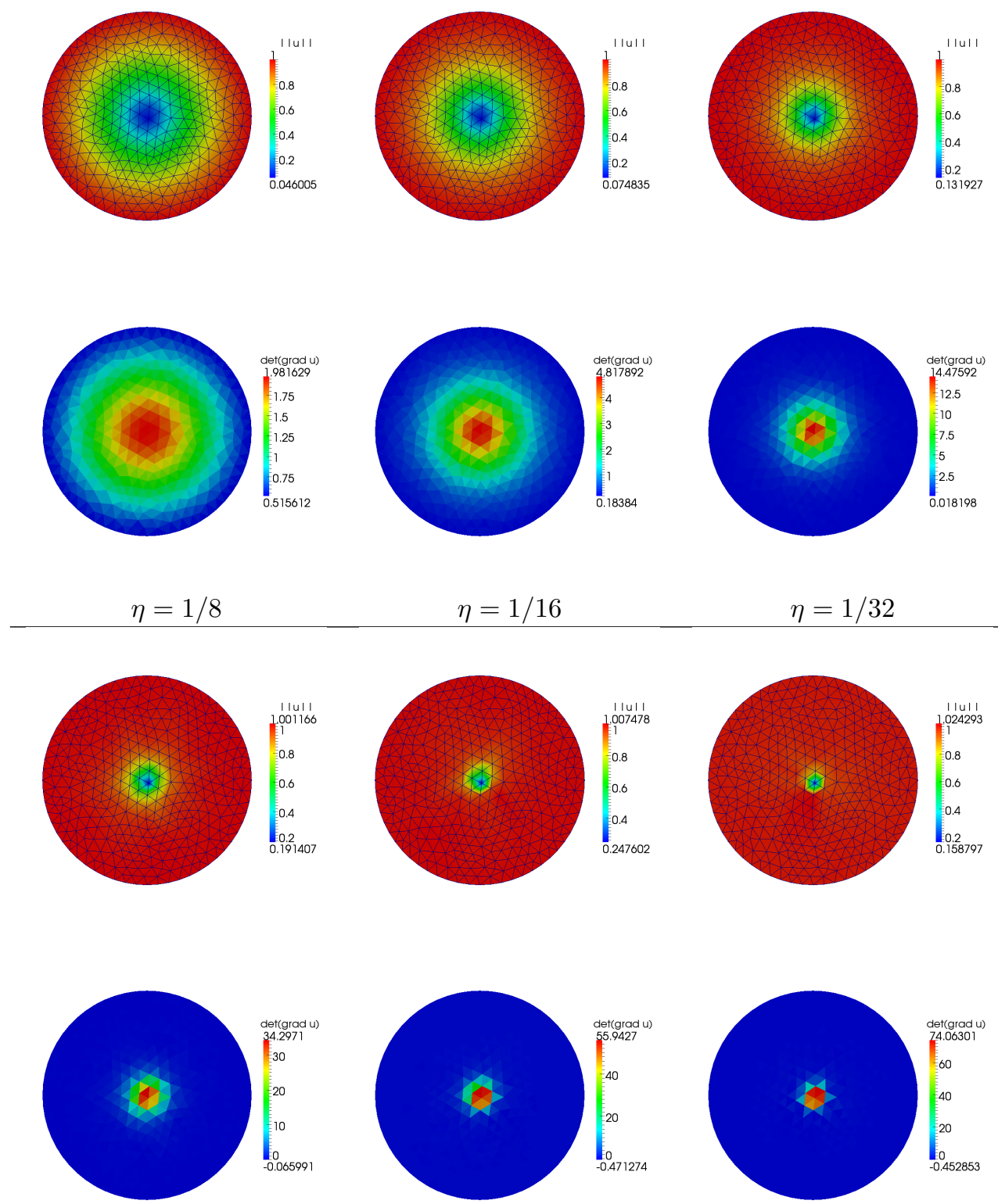

FIG. 17. Test problem with $f=\pi \delta_{(\mathbf{0})}$. Visualization of $\left\|\mathbf{u}_{\eta, h}\right\|_{0 h}$ (top) and $\operatorname{det} \nabla \mathbf{u}_{\eta, h}$ (bottom) after 100 iterations for various values of $\eta(h \simeq 0.0461, r=10, \delta=0.01)$.

mesh size (the same remark holds for $\operatorname{det} \mathbf{p}_{h}$, not represented here). Not imposing Dirichlet boundary conditions at the point $(0,0)$ is crucial to making the numerical algorithm converge. 

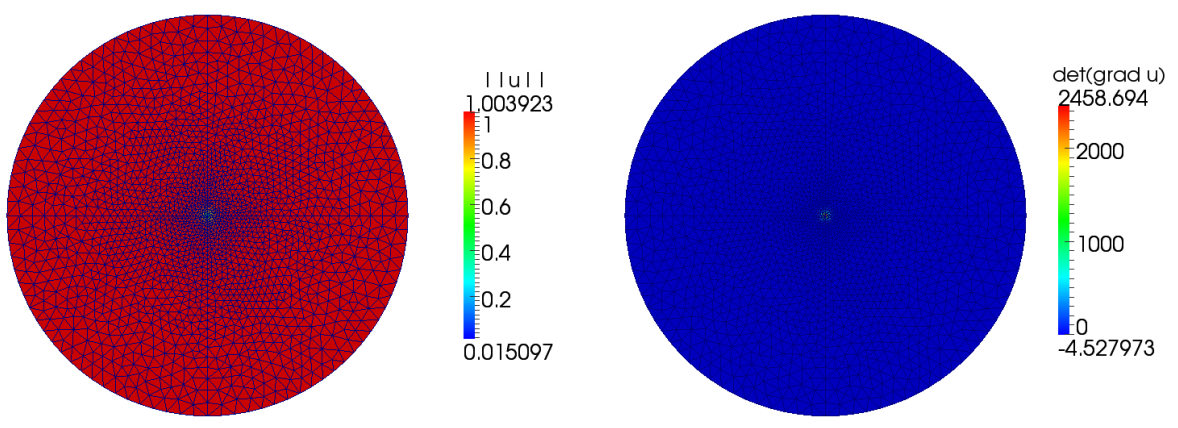

FIG. 18. Test problem with $f=\pi \delta_{(\mathbf{0})}$. Visualization of $\left\|\mathbf{u}_{\eta, h}\right\|_{0 h}$ (left) and $\operatorname{det} \nabla \mathbf{u}_{\eta, h}$ (right) after 100 iterations. $(\eta=1 / 64, r=100, \delta=0.0025)$. For this computation we have used a mesh locally refined around $(0,0)$, so that $\min _{K} h_{K}=0.0001$, while $\max _{K} h_{K}=0.005$.
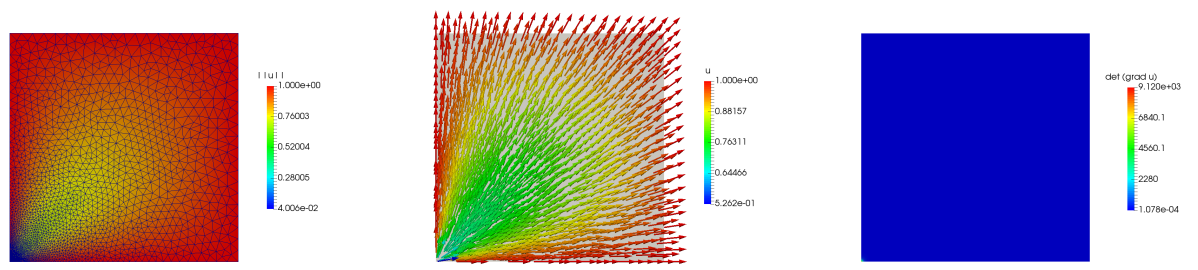

FIG. 19. Test case on the L-shaped domain. Visualization of the approximate solution obtained with the augmented Lagrangian $(h \simeq 0.01)$. Left: norm $\left\|\mathbf{u}_{h}\right\|_{2} ;$ middle: vector field $\mathbf{u}_{h} ;$ right: Determinant $\operatorname{det} \nabla \mathbf{u}_{h}$.

Acknowledgments. The authors thank Prof. B. Dacorogna (EPFL) for suggesting the investigation of this problem and some test problems of interest, Prof. M. Picasso (EPFL) for helpful comments and discussions, and the anonymous referees for constructive comments.

\section{REFERENCES}

[1] J. Bourgain and H. Brezis, Sur l'équation div $u=f$, C. R. Math. Acad. Sci. Paris, 334 (2002), pp. 973-976.

[2] J. Bourgain and H. Brezis, On the equation div $Y=f$ and application to control of phases, J. Amer. Math. Soc., 16 (2003), pp. 393-426.

[3] S. Boyd, N. Parikh, E. Chu, B. Peleato, and J. Eckstein, Distributed optimization and statistical learning via the alternating direction method of multipliers, Found. Trends Mach. Learn., 3 (2011), pp. 1-122.

[4] A. Caboussat, On the numerical solution of the Dirichlet problem for the elliptic $\left(\sigma_{2}\right)$ equation, in Modeling, Simulation and Optimization in Science and Technology, ECCOMAS, Springer, Dordrecht, 2014, pp. 23-40.

[5] A. Caboussat and R. Glowinski, Regularization methods for the numerical solution of the divergence equation $\nabla \cdot \mathbf{u}=f$, J. Comput. Math., 30 (2012), pp. 354-380.

[6] A. Caboussat and R. Glowinski, A numerical algorithm for a fully nonlinear PDE involving the Jacobian determinant., in Numerical Mathematics and Advanced Applications- 
ENUMATH 2013, Lect. Notes Comput. Sci. Eng. 103, Springer, Cham, 2015, pp. 143-151.

[7] A. Caboussat and R. Glowinski, A penalty-regularization-operator splitting method for the numerical solution of a scalar Eikonal equation, Chin. Ann. Math. Ser. B, 36 (2015), pp. 659-688.

[8] A. Caboussat, R. Glowinski, and V. Pons, An augmented Lagrangian approach to the numerical solution of a non-smooth eigenvalue problem, J. Numer. Math., 17 (2009), pp. 326.

[9] A. Caboussat, R. Glowinski, and D. C. Sorensen, A least-squares method for the solution of the Dirichlet problem for the elliptic Monge-Ampère equation in dimension two, ESAIM Control Optim. Calc. Var., 19 (2013), pp. 780-810.

[10] L. A. Caffarelli And R. Glowinski, Numerical solution of the Dirichlet problem for a Pucci equation in dimension two. Application to homogenization, J. Numer. Math., 16 (2008), pp. $185-216$.

[11] G. Carlier and B. Dacorogna, Résolution du problème de Dirichlet pour l'équation du Jacobien prescrit via l'équation de Monge-Ampère, C. R. Math. Acad. Sci. Paris, 350 (2012), pp. 371-374.

[12] P. G. Ciarlet, Basic error estimates for elliptic problems, in Handbook of Numerical Analysis, Vol. II, P. Ciarlet and J. Lions, eds., North-Holland, Amsterdam, 1991, pp. 17-351.

[13] R. Coifman, P. L. Lions, Y. Meyer, And S. Semmes, Compacité par compensation et espaces de Hardy, Séminaire Equations aux dérivées partielles (Polytechnique) (1989-1990), pp. 18 .

[14] M. G. Crandall, H. Ishit, and P.-L. Lions, User's guide to viscosity solutions of second order partial differential equations, Bull. Amer. Math. Soc. (N.S.), 27 (1992), pp. 1-67.

[15] G. Cupini, B. Dacorogna, And O. Kneuss, On the equation det $\nabla u=f$ with no sign hypothesis, Calc. Var. Partial Differential Equations, 36 (2009), pp. 251-283.

[16] B. Dacorogna, R. Glowinski, Y. Kuznetzov, and T.-W.Pan, On a conjugate gradient/Newton/penalty method for the solution of obstacle problems. application to the solution of an Eikonal system with Dirichlet boundary conditions, in Conjugate Gradient Algorithms and Finite Element Methods, R. G. M. Křížek, P. Neittaanmäki, and S. Korotov, eds., Springer-Verlag, Berlin, Heidelberg, 2004, pp. 263-283.

[17] B. Dacorogna and J. Moser, On a partial differential equation involving the Jacobian determinant, Ann. Inst. H. Poincaré Anal. Non Linéaire, 7 (1990), pp. 1-26.

[18] E. J. Dean and R. Glowinski, An augmented Lagrangian approach to the numerical solution of the Dirichlet problem for the elliptic Monge-Ampère equation in two dimensions, Electron. Trans. Numer. Anal., 22 (2006), pp. 71-96.

[19] I. Ekeland And R. TÉmam, Convex Analysis and Variational Problems, SIAM, Philadelphia, 1999, https://doi.org/10.1137/1.9781611971088.

[20] X. Feng, R. Glowinski, and M. Neilan, Recent developments in numerical methods for fully nonlinear second order partial differential equations, SIAM Rev., 55 (2013), pp. 205-267, https://doi.org/10.1137/110825960.

[21] X. Feng AND M. Neilan, Vanishing moment method and moment solutions for second order fully nonlinear partial differential equations, J. Sci. Comput., 38 (2009), pp. 74-98.

[22] X. FENG AND M. NeILAN, Finite element approximations of general fully nonlinear second order elliptic partial differential equations based on the vanishing moment method, Comput. Math. Appl., 68 (2014), pp. 2182-2204.

[23] M. Fortin and R. Glowinski, Augmented Lagrangian Methods: Application to the Numerical Solution of Boundary-Value Problems, North-Holland, Amsterdam, 1983.

[24] B. D. Froese and A. M. Oberman, Convergent finite difference solvers for viscosity solutions of the elliptic Monge-Ampère equation in dimensions two and higher, SIAM J. Numer. Anal., 49 (2011), pp. 1692-1714, https://doi.org/10.1137/100803092.

[25] R. Glowinski, Numerical Methods for Nonlinear Variational Problems, 2nd ed., SpringerVerlag, New York, 2008.

[26] R. Glowinski, Numerical methods for fully nonlinear elliptic equations, in ICIAM 07-6th International Congress on Industrial and Applied Mathematics, European Mathematical Society, Zürich, 2009.

[27] R. GLowinski, Variational Methods for the Numerical Solution of Nonlinear Elliptic Problems, SIAM, Philadelphia, 2015, https://doi.org/10.1137/1.9781611973785.

[28] R. Glowinski and A. Marrocco, Sur l'approximation par éléments finis d'ordre 1 et la résolution par pénalisation-dualité, d'une classe de problèmes de Dirichlet non linéaires, C. R. Acad. Sci. Paris Sér. A, 278 (1974), pp. 1649-1652.

[29] R. Glowinski AND A. MarRocco, Sur l'approximation par éléments finis et la résolution par pénalisation-dualité d'une classe de problèmes de Dirichlet non linéaires, Rev. Française

Copyright (c) by SIAM. Unauthorized reproduction of this article is prohibited. 
Automat. Informat. Recherche Opérationnelle Sér. Rouge Anal. Numér., 9 (1975), pp. 4176 .

[30] R. Glowinski, S. Osher, And W. Yin, eds., Splitting Methods in Communications and Imaging, Science and Engineering, Springer, New York, 2016.

[31] R. Glowinski And P. L. TAllec, Augmented Lagrangian and Operator-Splitting Methods in Nonlinear Mechanics, SIAM, Philadelphia, 1989, https://doi.org/10.1137/1. 9781611970838.

[32] H. Ishi AND P.-L. Lions, Viscosity solutions of fully nonlinear second-order elliptic partial differential equations, J. Differential Equations, 83 (1990), pp. 26-78.

[33] O. LAKKIS AND T. PRYER, A finite element method for nonlinear elliptic problems, SIAM J. Sci. Comput., 35 (2013), pp. A2025-A2045, https://doi.org/10.1137/120887655.

[34] J. NEČAS, Introduction to the Theory of Nonlinear Elliptic Equations, John Wiley \& Sons, Chichester, 1986.

[35] S. Osher And R. Fedkiw, Level Set Methods and Dynamic Implicit Surfaces, Springer-Verlag, New York, 2003.

[36] T. RivièRE AND D. YE, Resolutions of the prescribed volume form equation, NoDEA Nonlinear Differential Equations Appl., 3 (1996), pp. 323-369.

[37] J. A. Sethian, Fast marching methods, SIAM Rev., 41 (1999), pp. 199-235, https://doi.org/ 10.1137/S0036144598347059.

[38] D. C. Sorensen AND R. Glowinski, A quadratically constrained minimization problem arising from PDE of Monge-Ampère type, Numer. Algorithms, 53 (2010), pp. 53-66.

[39] X.-C. TAI, J. Hahn, And G. J. Chung, A fast algorithm for Euler's elastica model using augmented Lagrangian method, SIAM J. Imaging Sci., 4 (2011), pp. 313-344, https://doi. org $/ 10.1137 / 100803730$.

[40] C. WU AND X.-C. TAI, Augmented Lagrangian method, dual methods, and split Bregman iteration for ROF, vectorial TV, and high order models, SIAM J. Imaging Sci., 3 (2010), pp. 300-339, https://doi.org/10.1137/090767558.

[41] D. Ye, Prescribing the Jacobian determinant in Sobolev spaces, Ann. Inst. H. Poincaré Anal. Non Linéaire, 11 (1994), pp. 275-296.

Copyright (C) by SIAM. Unauthorized reproduction of this article is prohibited. 